\title{
AUTHOR INDEX*
}

ABC, 308, 314, 421, 423, 425

Abernathy, W, 318, 336

Abeysinghe, T, 115, 118

Abou Zaid, M, 931, 935, 937

Aboudha, PA, 366, 384

ABS, 301, 450, 457, 494

AC Pigou, 802

Accor, 181, 183, 184, 188, 751, 753, 754

Acosta-Gonzalez, G, 782

Adam, S, 37, 102, 139, 492

Adamowicz, J, 384

Adamowicz, WL, 367, 384

Adams, 318, 319, 336, 472-475, 488, 489, $506,511,519$

ADB, 742, 755, 756

AECOM, 446, 464

Agarwal, 140, 160, 163, 165, 548, 567

Agndal, H, 150, 153, 159, 161

Aguilar, F, 847, 851, 860, 864

Aguirre, SZ, 637

Ahmt, T, 545, 548, 568

Airey, D, 429, 438

Aislabie, CJ, 65

Aitken, B, 176, 188

Ajzen, I, 378, 381, 384

Akella, A, 868

Akinboade, OA, 631, 636

Álavarez-Gil, MJ, 150, 161

Albaum, G, 103

Aldenderfer, MS, 93, 101

Alexander, LM, 545, 567

Alexander, N, 153, 154, 161

Ali, S, 937

Allen, 277

Allen, K, 840

Allen, RC, 232, 233, 242, 243, 251

Allen Consulting Group, 463, 465

Almeida, VMC, 169
Alsarayreh, MN, 125, 132

Alston, J, 63, 64

Altinary, M, 545, 547, 568

Altinay, L, 150, 157, 161, 167

Altman, 335, 336, 797, 808

Alvares, C, 692, 694, 696, 697, 702

Álvarez-González, JA, 568

Amiti, M, 577, 600

Amorim, C, 150, 161

Anastassopoulos, G, 153, 161

Anderson, E, 141, 161, 165

Anderson, G, 69, 75, 82

Andersson, J, 260, 277

Andersson, P, 150, 161

Andreu, R, 168

Angle, PS, 688, 702

Anning, D, vi, 361, 388

Anon, 131, 132, 443, 815, 840

Antonucci, R, 349, 356

ANZECC, 434, 438

Apostolides, AD, 238, 251

Appleby, J, 291

Arabie, P, 95, 101, 103

Arbache, JS, 489, 678

Archer, 544-547

Arias-Gonzalez, JE, 782

Arima, T, viii, 26, 27, 885, 888, 894, 908, 910

Arlt, W, 304, 314

Armstrong, G, 102, 815

Arndt, SW, 577, 579, 600

Arnegger, J, 570

Arrow, KJ, 60, 61, 377, 383, 384

Artus, JR, 42, 61

Aryal, K, 847, 864

Ashley, C, 747, 758

Ashley, GA, 568

*Entries relating to institutions/organizations are listed in both Author Index and Subject Index. 
Ashworth, JS, 911, 937

Askitas, N, 287, 294

ASPE Research Brief, 294

ASTA, 192, 193, 196, 204

Atiyah, P, 387

Attfield, CLF, 75, 82

AusAID, 742, 744, 756

AusPost, 448, 465

Australian Bureau of Statistics (ABS), 300, 301, 314, 375, 448, 465

Australian Taxation Office (ATO), 449, 465

Aydin, N, 536, 540

Aylward, B, 435, 439, 819, 840

Ayuso, S, 847, 864

Azahari, O, 434, 439

Baade, R, 412

Baaijens, SR, 548, 568

Bacha, EL, 622, 636

Bain, J, 140, 161

Baird Review, 314

Bakas, F, 846, 848, 864

Baker, L, 275, 278, 287, 294

Bakkal, I, 61, 68, 69, 73, 82

Balaguer, J, 630, 636

Balassa, B, 575, 588, 589, 600, 601, 622, 623, 636

Baldwin, R, 577, 582, 600

Ball, D, 151, 161

Ballantyne, R, 262, 278, 874, 878, 882,883

Bandera, VN, 147, 161

Banegil, T, 846, 864

Bansal, S, 147, 164

Banzhaf, 386, 916, 918, 937

Bao, 440, 726, 736, 740

Barbaro-Forleo, G, 866

Barbier, E, 810

Barendregt, A, 940

Bar-Hen, A, 296

Barnett, 57, 61, 847, 864

Barras, R, 274, 277

Barro, SC, 385

Barron, 296

Barten, AP, 44, 52, 56, 57, 60, 74, 82

Bastakis, C, 202, 205

Bastianoni, S, 841

Bateman, IJ, 364, 384, 388, 448, 465

Battalio, R, 274, 278, 330, 336
Baum, T, 153, 161, 222, 548, 566, 570

Baumol, W, 16, 29, 264, 277, 778, 782

Bayfield, NG, 806, 808

BBC, 284, 294

Beale, D, 462, 466

Beamish, PW, 138, 161, 263

Bechdolt, B, 238, 252

Becherel, L, 677, 681

Becker, E, 828, 840

Beckermann, W, 143, 161

Beckmann, I, 341, 358

Beer, C, 937

Belloumi, M, 631

Belt, M, 385

Benedetto, G, 783

Benedictis, 588, 601

Bennett, J, 370, 388, 674, 679, 873, 882

Bergeron, 866

Bergson, A, 59, 62, 420, 439

Bergstrom, JC, 545, 547, 568

Berndt, ER, 50, 62, 74, 82

Berneron-Couvenhes, M-F, 339, 340, 347,356

Bernhardt, JM, 297

Bernheim, BE, 61

Berno, T, 751, 756

Bertazzoni, S, 358

Bethencourt-Cejas, M, 568

Betts, K, 315

Bezbaruah, MP, 706, 722

Bhagwati, JN, 37, 62, 601, 623, 636

Biederman, P, 237, 238, 252

Bieger, T, 206, 209, 210, 221, 413, 603, 680, 681

Biehn, T, 353, 357

Bien, A, 846, 864

Bierman, H, 412

Billiet, J, 104

Billington, N, 140, 162

Bingham, G, 363, 384

Binning, C, 914, 937

Birch, DW, 285, 294

Birrell, B, 308, 315

Bishop, RC, 369, 384

Bisigniani, G, 209, 222

Björkman, I, 149, 150, 152, 162

Blackorby, C, 49, 62

Blackwell, B, vi, 16, 384, 386, 443, 444, 447, 448, 466

Blake, A, 472-480, 482-485 
Blashfield, RK, 101

Blinder, AS, 577, 600

Blomstermo, A, 150, 151, 162

Blundell, RW, 69, 75, 82

Blust, 192, 205

Blyth, E, 284, 294

Boardman, A, 395-398, 400, 401, 403, 410, 412

Bockstael, N, 873, 882

Bohdanowicz, P, 752, 757, 847, 864

Bohlen, G, 865

Bohlmann, HR, 474, 482, 483, 489

Bond, L, 361, 388, 466, 937

Bonham, C, 247, 252

Bonn, MA, 548, 568

Bookman, KR, 285, 294

Bookman, MZ, 285, 294

Booz, 265, 277

Böhringer, C, 506, 519

Boskovic, D, 357

Bostrom, N, 817, 840

Boumans, R, 937

Boumans, RMJ, 388, 938

Bowitz, E, 545, 568

Bowker, JM, 386

Bowles, J, 6

Bowles, S, 29

Boxall, 370

Boxall, PC, 384

Boyd, J, 916, 918, 937

Boyle, KJ, 384

Boyle, S, 383

Boylem, S, 295

Bozec, YM, 768, 782

Bracalente, B, 270, 277

Bradley, M, 868

Bradly, AP, 752, 757

Braimoh, LA, 631, 636

Bramwell, B, 794, 798, 808

Branger, B, 295

Brau, R, 632, 633, 637, 640

Braun, B, 264, 277

Braun, BM, 545, 547

BREA, 355, 356

Breda, Z, v, vii, 8, 21, 137, 150, 154, 155, 158, 159, 162, 170, 683, 704

Breiter, M, 402, 413

Breusch, TS, 112, 118

Brida, JG, 355, 357, 631, 637, 640

Britton, S, 747, 757
Brody, M, 384

Broe, ME, 295

Bromley, D, 384

Bromley, DW, 384, 882

Brown, JAC, 58, 62

Brown, K, 270, 277

Brown, L, 102

Brown, R, 882

Brown, RPC, 883

Brown, TC, 383-385

Bruno, M, 622, 637

Brunt, P, 570

Bryden, JM, 647, 661, 678, 788, 808

Buchta, C, 96, 97, 101

Buckley, PJ, 141, 147, 148, 152, 153, 162

Buckley, R, 846, 849, 863-865

Budiani-Saberi, D, 284, 294

Budowski, G, 432, 439

Buhalis, D, 194, 202, 205, 221, 222

Bull, A, vi, 13, 339, 340, 342, 344, 351, 353, 357, 359, 395, 403, 412, 568, 645, 646, 658, 670, 678, 808

Bundorf, MK, 294

Bunting, J, 868

Buol, SW, 902, 908

Burgan, B, 394, 399, 407, 412, 446, 465

Burgess, C, 153, 162

Burgman, M, 914, 937

Burkart, AJ, 577, 601

Burns, AF, 228, 252

Burns, P, 648, 678

Burton, R, 686, 702

Burton, RCJ, 805, 809

Busby, G, 570, 789, 809

Buse, A, 57, 62

Business Research and Economic Advisors (BREA), 357

Busuttil, L, 218, 223

Butler, RW, 10, 29, 208, 212, 222, 225-230, 243, 248, 250, 252, 655, 659, 678, 686, 702

Butler, JR, 799, 809

Butler, R, 205

Cain, LP, 249, 253

Calvert, EO, 758

Calvet, AL, 140, 162

Calvo, P, 602, 775, 783

Cambre, B, 104

Campbell, JB, 101 
Campbell, R, 784

Campbell-Hunt, C, 145, 162

Canada, 316

Canfield, C, 637

Cantavella-Jordá, M, 630, 636

Caralt, J, 279

Carbajal, E, 637

Cardone-Riportella, C, 161

Carey, G, vii, 20, 34, 62, 619, 641

Caric, H, 355, 357

Carlos, A, 74, 83, 170, 572, 704

Carlsen, J, 439, 874, 881, 882

Carr, DL, 188

Carson, RT, 368, 387, 873, 882

Casson, M, 141, 147, 162

Castro, EA, vii, 19, 543, 572

Čavlek, N, vi, 9, 191, 202, 205, 206

Ceballos-Lascuráin, H, 829, 840

Centre d'Etudes Prospectives et d'Informations Internationales (CEPII), 601

Centre for Agricultural and Regional Economics, 874, 875, 882, 883

CEPII, 590-592, 595-599, 602

Cerina, F, 627, 637

CES, 692, 702

Cesar, H, 920, 937

Cesare, FDi, 358

Chafe, Z, 849, 859, 864

Chaiken, S, 378, 385

Chakravorty, U, 492, 572

Chalfant, JA, 59, 62

Chamorro, A, 846, 864

Champ, PA, 384

Chang, T, 277

Chao, C-C, viii, 19, 20, 605, 615-618

Chao, Y, 862, 864

Chapman, DJ, 375, 385

Charlier, J, 343

Chattopadhyay, K, v, 7, 121, 124, 125, 132, 133, 722

Chaudhary, P, 864

Chen, CF, 632, 637

Chen, JJ, 153, 155, 157-159, 162, 164

Chen, GM, 190

Chen, M, 638

Chenery, HB, 622, 637

Chetty, 145, 162

Cheung, P, 321, 337

Cheyne, J, 847, 864
Chin, CBN, 345, 351, 357, 358

China Daily, 184, 188

China National Tourism Administration, $173,174,184,188,190$

China Tourist Hotels Association, 188

Chiou-Wei, SZ, 632, 637

Chirieleison, C, 277

Cho, M, 153, 162

Choi, H, 294

Chou, FS, 118

Choy, DJL, 791, 794, 809

Christ, C, 846, 864

Christensen, LR, 53, 62

Chryssohoidis, G, 847, 856, 866

Chun, KT, 94, 101

Cicic, M, 151, 168

Cifuentes, M, 440

Claessens, S, 150, 162

Clancy, M, 789, 809

Clark, C, 4, 29

Clark, E, 384

Clark, GC, 814, 840

Clark, K, 318, 336

Clark, T, 151, 162

Clarke, H, 412, 423, 429, 807

Clarke, WC, 757

Clavé, S, 318, 326, 327, 331, 336

Claver, E, 168

Clawson, M, 260, 277

Clemen, B, 868

Clements, KW, 58, 65

CLIA, 341, 345, 348, 349, 357

Clifford, W, 868

COAG, 311, 315, 444, 465

Coase, R, 141, 162, 803, 809

Cockerell, N, 500, 519

Coe, NM, 150, 163

Coenen, M, 68, 70, 71, 73, 83

Cogneau, D, 487, 490

Cohen, S, 232, 252

Colby, B, 570

Cole, DN, 810

Collier, 822, 836, 840, 853, 865

Collins, D, 88, 101

Colmey, J, 834, 840

Conaghan, A, 846, 865

Conklin, DW, 180, 181, 189

Connell, J, 154, 163, 282, 284-286, 288, 294, 570 
Contractor, FJ, 149, 154-156, 158, 159, 163

Cook Islands Statistics Office, 749, 757

Cook Islands Tourism Corporation, 748, 757

Cook, J, 757

Cooper, C, 197, 205, 278, 484, 491, 545, $568,576,603$

Cooper, P, 940

Cooper, RJ, 57, 59, 62

Cooper, W, 384

Copeland, BR, 20, 29, 40, 62, 606, 609, $612,617,624,637$

Cordell, HK, 568

Corden, WM, 609, 617

Cork, S, 937

Cornelissen, S, 576, 601, 846, 865

Cortés-Jiménez, I, 43, 62, 70, 75, 83, 632, 635, 637, 639

Cortez, H, 784

Cossignani, M, 277

Costa Carlos, v, vii, 8, 21, 137, 170, 543, $572,683,704$

Costa, C, 8, 19, 148, 163

Costanza, R, 362, 384, 385, 388, 914, 915, 918, 921, 937, 938

Council of Australian Governments (COAG), 315, 465

Courvisanos, J, 774, 775, 783

Coviello, N, 138, 145

Cowling, RM, 938

Coxhead, I, 665

Craig-Smith, S, 315, 749, 759

Crampon, LJ, 230-233, 252

Crandall, K, 570

Crask, M, 90, 101

Crilley, G, 439

Crompton, JL, 729, 732, 739

Crouch, G, 674, 680

Crouch, GI, 238, 252, 548, 569

Crouthamel, J, 433, 439

Cruise Lines International Association (CLIA), 340, 357

Culberston, K, 805, 809

Cullen, R, 434, 439

Cunningham, P, 899, 908

Curry, K, 702, 868

Curtin, J, 789, 809

Curtis, J, 434, 435, 439
Da Fonseca, GAB, 841

Daelemans, R, 295

Daily, GC, 914, 938

Dalgic, T, 150, 166

Daniel, P, 83

Daniels, P, 150, 163

Daniels, MJ, 548, 568

Danigelis, NL, 937

Dantas, N, 696, 702

Darder, FL, 150, 153, 155, 157, 159, 168

d'Arge, R, 937

Darling, J, 846, 847, 865

Dasgupta, A, 412

Davé, U, 153, 163

Davidson, D, 854, 865

Davidson, J, 76, 83

Davidson, M, 304, 312, 315

Davies, L, 679

Davies, REG, 233, 237, 243, 252

Davis, D, 550, 928, 930, 931, 938

Dawson, D, 283, 294

De la Cruz, JL, 631

Deardorff, A, 579, 601

Deaton, A, 44-46, 50, 54-56, 58, 59, 62, $63,74,83$

Deegan, J, 70, 83

Deery, M, 490

Dekker, FW, 296

Delacy, T, 386

Delmonico, F, 294

Demunter, C, 670-672, 678

Denstadii, J, 26, 29, 883

Department of Business, Economic

Development \& Tourism, 541

Department of Environment, Climate Change and Water, $878,879,881,883$

Department of Finance, 408, 412

Department of Infrastructure and Planning, 465

Department of Natural Resources and Environment, 465, 541

Department of Statistics, 105, 118, 760

Department of Sustainability and

Environment, 465

DERM, 460, 465

Derviş, K, 506, 519

Desvousges, WH, 386, 387

Dev, CS, 156, 165, 163

Devesa, 277, 637

Dhaw, N, 132 
Diamantis, D, 167, 602

Diamantopoulos, A, 854, 865

Diamond, PA, 369, 385

Díaz-Pérez, FM, 548, 568

Dibb, S, 90, 101

Dickey, DA, 628, 637

Dickey-Fuller, 628

Dickinson, J, 937

Diedrich, A, 355, 357

Dieke, PUC, 647, 678

Diewert, WE, 45, 53, 59, 63, 76, 83

Dillman, DA, 373, 381, 385

Dimitriadou, E, 101

Dimou, I, 153, 155, 157-159, 162, 164

DIP, 446, 465

Ditton, R, 363, 387

Divisekera, S, v, 5, 6, 33, 40, 42, 43, 63, 66-68, 70-74, 79, 80, 83, 85

Dixit, AK, 37, 63, 264, 277, 579, 601

Dixon, JA, 928, 932, 938

Dixon, P, 506, 519

Doganis, R, 209, 213, 215, 222

Doi, T, 898-900, 908

Dolado, JJ, 628, 637

Dolnicar, S, v, 6, 7, 87, 88, 90, 92, 95-98, 100-102, 104

Dong, H, 665, 680

Donnelly, M, 868

Dornbusch, R, 584, 585, 601, 602

Douglas, N, 53, 55, 59, 234, 349, 354, 355, 357,625

Dovers, S, 445, 466

Dowling, R, 349, 357, 358

Downer, M, 805, 809

Downward, PM, 566, 568

Doxey, G, 684, 702

Dragin, A, 355, 357

Dragun, AK, 23, 24, 765, 766, 769, 783,785

Dreike, O, 863, 865

Driel, JV, 52, 64

Driml, S, 25, 26, 849, 865, 871, 872, 874, $878,880,881,883,884$

Dritsakis, N, 634, 637

Drueke, TB, 284, 296

D'Sa, E, 696, 702

DSE, 444, 465

Duhs, A, vi, 12, 299, 300, 304, 316

Dunford, RW, 386
Dunning, JH, 139-141, 147, 153, 154, 158, $164,181,189,578,601$

Durbarry, R, 62, 75, 84, 489, 632, 637

duVair, PH, 377, 387

Dwyer, L, 30, 64, 205, 272, 277, 354, 357, 406, 407, 409, 412, 430, 433, 439-441, 473, 475, 477, 482, 487-490, 493, 496, 499, 500, 505, 510, 519, 520, 540, 545, $547,548,568,664,669,678,770,783$, $809,883,884$

Dwyer, W, 272, 277, 439, 519, 678

Dybedal, P, 330, 336

Eagly, AH, 378, 382, 385

Easterley, W, 836, 840

Ebel, R, 237, 252

Echeverria, J, 840

Econtech, 473, 477, 490

ECORYS, 196, 198, 205

Ecotourism Australia, 773, 783, 848, 865

Edmonds, C, 252

Edvardsson, B, 150, 164

Edvinsson, L, 164

EEAA, 912, 925, 935, 938

Eekeren, M, 70, 71, 73, 83

Egoh, B, 914, 938

EIU, 198, 205

Ekeledo, I, 149, 150, 164

Elbe, J, 150, 153, 159, 161

Elliott, C, 244, 252, 937

El-Mourhabi, J, 167, 602

El-Taha, M, 358

Emery, RF, 621, 622, 637

Endo, K, 576, 601

Engle, RF, 112, 118, 628, 638

Englin, JE, 369, 385

English, BG, 103

English, V, 284, 294

Engwall, L, 152, 164

Enhance Management, 849, 865

Ennew, C, 744, 757

Epstein, R, 276, 277

Erdem, T, 270, 277

Eric, N, 123, 132

Erikson, L, 545, 548, 568

Ernest \& Young Global, 187, 189

Eroglu, D, 297

Erramilli, MK, 149-152, 154, 159, 163, 165

Esparon, M, viii, 25, 845, 868 
Essex, S, 868

Eugenio-Martin, JL, 489, 633, 638

European Commission, 215, 217, 222, 682

EUROSTAT, 519, 520, 497, 540

Eurostat, 578, 601

Eurostat/OECD, 580

Eusébio, vii, 19, 543, 545, 547, 548, 550, $568,570,572$

Everitt, BS, 97, 102

Fairweather, J, 848, 859, 862, 865

Fakeye, PC, 732, 739

Falco-Mammone, F, 849, 867

Fallas, H, 282, 294

Fallon, D, 277

Farber, S, 385, 937, 938, 940

Farley, J, 937

Farrand, A, 284, 294

Fayall, A, 138, 165

Fayed, H, 576, 601

Fayissa, B, 633, 638

Feenstra, RC, 579, 601

Fei, B, 65

Feldstein, MS, 61

Fenell, D, 886, 908

Ferguson, L, 486, 490

Ferjanić Hodak, D, 205

Fernández, J, 279

Ferrucci, L, 277

Fiji Bureau of Statistics, 750, 757

Filippaios, F, 161

Finke, R, 63

Finn, A, 270, 277

Firmin, R, 863, 866

Fishbein, M, 378, 384

Fisher, AC, 387, 873, 883

Fisher, B, 914, 916, 917, 937, 938, 940

Fisher, S, 601

Flachaire, E, 851, 853, 865

Flahault, A, 296

Flash Eurobarometer, 192, 328

Fleischer, A, 545, 547, 569

Fleming, DK, 213, 222

Fletcher, D, 548, 571

Fletcher, J, 205, 544-548, 567-569, 576, 582,601

Flood, LR, 57, 59, 63

Foley, M, 262, 277

Folke, C, 385, 915, 916, 919, 920, 939

Font, X, 846, 864, 865
Fontenot, R, 868

Formann, AK, 95, 102

Forsgren, M, 145, 165

Forsyth, K, 855, 865

Forsyth, P, 205, 223, 277, 354, 357, 406, 407, 412, 430, 439, 440, 489, 490, 519, $520,545,568,678,783,797,809,883$

Fossati, A, 767, 769, 770, 783, 784

Foster, G, 306, 315

Fourcade, J, 295

Frank, RE, 100, 102

Fraser, I, 73, 83

Frechtling, D, 498, 523, 540, 548, 569

Freckleton, R, 283, 294

Fredline, L, 490

Freeman, D, 545, 547, 569

Fresenmair, DR, 545, 547, 569

Freudenburg, W, 854, 865

Friedman, TL, 818, 840

Frisch, R, 49, 63

Frissell, SS, 810

Frochot, I, 90, 102

Fujii, E, 70, 73, 83, 427, 428, 439

Fuller, WA, 628, 637

FVW, 197, 206

Fyall, A, 205, 278, 279, 337

Gallant, AR, 53, 63

Galle, J, 297

Galt, M, 492

Gamage, A, 545, 547, 548, 571

Gamage, AR, 124, 132

Gamba, RJ, 103

Gandolfi, F, 150, 165

Gangadharan, L, 409, 413

Ganz, W, 150, 165

Garcia, C, 283, 295

García-Falcón, MJ, 206

Gardiner, S, 315

Gardner, J, 294

Garrison, CB, 545, 565, 569

Garrod, B, 138, 165, 278, 279, 337

Gartner, WC, 645, 646, 679, 732, 739

Gatignon, H, 141, 161, 165

Gay, D, 747, 757

Gayer, DE, 937

Geddes, R, 270, 277

Gehlhar, M, 83

George, B, 289, 295

Georgiou, S, 940 
Getz, D, 260, 277

Geyikdagi, NV, 152, 153, 162

Geyskens, E, 82

Ghartey, EE, 631, 638

Ghemawat, P, 107, 118, 814, 840

Giarini, O, 37, 63

Gibson, D, 755, 757

Gil, J, 851, 866

Gilbert, A, 939

Gilbert, D, 205, 568

Gilbert, DC, 578, 601

Giles, JA, 623, 628, 638

Gillespie Economics, 849, 866

Gillespie Economics and BDA Group, 875-877, 883

Gillham, J, 489

Gilpin, R, 788, 789, 809

Ginman, PJ, 42, 64

Glaister, S, 413

Glenn, LM, 937

Goeldner, CR, 193, 206, 727, 739

Gold Coast City Council (GCCC), 16, 17, $385,445,447,460,464-466$

Goldberger, AS, 44, 51, 59, 63

Goldman, SM, 49, 63

Goldstein, M, 43, 63

Gómez, CM, 627, 638, 639

González, M, 639

Goodall, B, 847, 867

Goodman, SL, 364, 385

Goodman, WC, 235, 238, 241, 242, 252

Gooroochurn, N, 474, 480, 489, 490, 610,617

Gorman, WM, 57, 63, 211, 222

Gort, M, 228, 252

Gössling, S, 766-769, 783

Government of Arunachal Pradesh, 708, $715,718,722$

Government of Goa, 684, 692, 693, 695, 698-700, 702

Government of India, 125, 127, 128, 133, 684, 690-692, 695, 702, 713, 716, 722

Government of Samoa, 744, 757

Gowdy, J, 420, 439

Gracia, A, 866

Graddy, E, 228, 253

Graham, A, 218, 219, 222, 223

Granger, CWJ, 118, 627-629, 631, 634-636, 638

Grasso, M, 385, 937
Gray, HP, 28, 35-37, 42, 43, 29, 63, 245,252

Green, EP, 939

Green, R, 57, 63, 64

Green, RJ, 769, 774, 783

Greenberg, D, 412

Greene, WH, 368, 385, 923-925, 938

Greenwood, C, 492

Griest, DA, 805, 809

Griffith, A, 785

Griffith, DA, 166

Gris, JC, 284, 295

Gronau, R, 236, 252

Groot, 363, 385, 916, 937, 938

Gross, S, 357

Grossman, GM, 577, 579, 601

Grün, B, 95, 102

Gu, HM, 8, 171, 175, 177, 179, 189, 190

Gui, L, 343, 357

Guilding, C, 868

Gulliksen, V, 339, 340, 357

Guo, J, 526, 540

Gupta, S, 289, 295

Gyimothy, S, 729, 739

Gyuris, E, 25, 845, 869

Hadley, N, 363, 387

Hagedorn, K, 29

Haites, EF, 238, 252

Hajkowicz, SA, 370, 385

Hale, T, 384

Haley, D, 865

Haley, RJ, 90, 102

Hamblin, R, 283, 295

Hamilton, 277

Hampton, A, 162

Han, Z, 68, 73, 84

Hancock, D, 284, 286, 295

Hanemann, MW, 369, 386

Hanemann, W, 851, 866

Hanemann, WM, 375, 385

Hanley, N, 368, 386

Hannon, B, 385, 937

Hanrahan, J, 846, 865

Hansen, C, 545, 569

Hansen, E, 867

Hanson, F, 285, 295

Hanson, GH, 579, 601

Hansson, A, 147, 165

Harberger, AC, 506, 519 
Hardeman, R, 295

Hardin, G, 792, 809

Harding, P, 693, 702

Harman, F, 408, 412

Harrington, J, 548, 568

Harris, JR, 611, 617

Harris, P, 170

Harris, TR, 571

Harrison, A, 295

Harrison, AE, 176, 188

Harrison, D, 22, 23, 68, 648, 662, 679, $741,744,748-750,755-757,760$

Harrison, MJ, 41-43, 64, 69, 84

Hartley, K, 401, 413, 418, 429, 431, 439, 441

Harvard Medical Letter, 282, 286, 288, 295

Haskel, JE, 189

Hassid, J, 150, 165

Hausken, K, 125, 132

Hausman, JA, 369, 385

Hauteserre, 269, 277

Hawaii State Department of Business, 523,540

Hawkins, CJ, 412

Hawkins, JP, 929, 931, 935, 938

Hay, B, 489

Hayden, G, 384

Hayek, FA, 141, 165

Hayuth, Y, 213, 222

Hazari, BR, 606, 617, 623, 625, 626

Healy, E, 315

Heaney, L, 499, 520

Hebelein, TA, 932, 939

Heckscher, E, 37, 165

Heckscher-Ohlin, 139, 579

Hedin, K, 147, 165

Heerden, 474

Hefner, F, 412

Heidt, T, 863, 866

Hein, L, 918, 938

Helalat, MS, 132

Hellman, P, 150, 152, 165

Helpman, E, 37, 64

Hemstra, S, 428, 439

Hendee, JC, 805, 806, 809

Hennart, J-F, 141, 147, 165, 166

Hensher, DA, 923, 925, 938

Henten, A, 150, 166

Herath, G, 434, 435, 439
Hernández, JM, 639

Hernández-Martín, R, 623, 626, 638

Hernansanz, C, 150, 169

Herrero, L, 275, 277

Herrlinger, S, 297

Hertel, T, 506, 520

Heung, VS, 281, 286, 295

Hewings, GJD, 490

Hicks, JR, 44, 64

Higginbottom, K, 441, 769, 774, 775, 783,784

Hillel, O, 864

Hitch, TK, 249, 253

Hjalager, AM, 137, 166, 277, 265, 576, 577,601

Hjerpe, EE, 548, 569

Ho, T, 783

Hoban, T, 868

Hobson, JP, 342, 344, 357

Hoehn, JP, 381, 386

Hohl, A, viii, 25, 813, 842

Hole, FD, 908

Hollard, G, 851, 853, 865

Hölldobler, B, 834, 842

Holling, CS, 919, 938

Holm, BD, 145, 162

Holm, U, 165

Holz, CA, 172, 173, 189

Holzner, M, 634, 638

Honey, M, 788, 789, 809, 817-819, 824, 826-828, 832, 834, 836, 840, 846, 866, $886,887,908$

Hong, C, 490

Hong, JH, 166

Hooper, N, 429, 439

Hopkins, GL, 297

Hopkins, L, 282, 286, 295

Hoque, S, 519, 520, 883

Horowitz, MD, 286, 295

Horváth, E, 548, 569

Hospital, JD, 386

HOTELS, 179, 189

Houston, JR, 323, 362, 386

Houthakker, HS, 43, 49-51, 64

Howarth, R, 439

Hsu, C-C, 163

$\mathrm{Hu}, \mathrm{SW}, 617$

Hubert, L, 95, 101

Hudspeth, T, 937

Hughes, J, 249, 253 
Hughes, HL, 546, 569

Hughes, M, 433, 439

Hui, CH, 102

Hummels, D, 577, 587, 601

Hung, K, 347, 357

Hunt, S, 466

Husein, J, 631, 638

Huybers, T, 674, 679

Huysmans, K, 284, 295

Hymer, SH, 140, 166

IAAPA, 317, 336

Ibenholt, K, 545, 568

Ichiki, S, 895, 903, 904, 908

Idda, L, 775, 781, 783

IDP, 310, 315, 603

IFAD, 815,840

Ihalanayke, R, 132

Iijima, Y, 909

INE, 548, 549, 551, 569

Inskeep, E, 685, 702

International Monetary Fund (IMF), 589, 590, 600, 602, 621, 638, 648, 649, 679

Intralawan, A, 387

IRTS, 494, 496-498, 520

Isaacs, JC, 772, 774, 783, 832, 840

Ishigaki, A, 908

Ishihara, T, 899, 908

Ishii, J, 601

Ismail, J, 428, 439

Iso-Ahola, 729, 732, 739

Ivarez-Albelo, CD, 623, 626, 638

Iversen, C, 140, 166

Iverson, TJ, 548, 570

Jacobs, R, 294

Jafari, J, 103, 679, 819, 840

Jaffry, SA, 385

Jäger, S, 194, 206

Jago, L, 490

Jain, A, 774, 775, 783

Jakobsson, KM, 23, 24, 765, 766, 769, 783,785

Jang, S, 638, 732

Jansen, M, 150, 162

Jansen-Verbeke, M, 260, 262, 278

Japan Ecotourism Society, 887, 909

Japan Travel and Tourism Association, 888, 908

Japan Travel Bureau (JTB), 247, 253, 909
Jarvis, J, 750, 758

Jarvis, N, 862, 866

JATA-NET, 246, 247, 253

Jauhari, V, 847, 866

Javalgi, RRG, 150, 166

Jawabreh, OAA, 132

Jenkins, CL, 683, 702

Jensen, S, 545, 569

Jessamy, V, 940

Jesupatham, S, 698, 703

Jiao, Y, 359

Job, H, 570

Johansen, L, 506, 519, 638

Johanson, J, 142-145, 147, 165, 166

Johns, N, 729, 739

Johnson, C, 153, 166

Johnson, FR, 370, 386

Johnson, RL, 545, 547, 569, 822, 833, 874

Jones, C, 500, 501, 519

Jones, CA, 295

Jones, L, 569

Jones, P, 153, 157, 166

Jones, PT, 837

Jones, RW, 577, 579, 602

Jones, T, 268, 278

Jong, 487, 492

Jonker, S, 868

Jorgenson, DW, 62

Jovanovic, B, 228, 253

Jovicic, D, 357

Judge, RP, 369, 386

Jurowski, C, 732, 740

Kadt, 756

Kahle, L, 867

Kahn, ME, 287, 295

Kahn, RF, 547

Kahneman, D, 6, 29

Kaiser, K, 96

Kaizu, Y, 908

Kalafatis, S, 170

Kaldor, N, 141, 166

Kaldor-Hicks, 418, 419, 437

Kamakura, W, 97, 100, 103

Kamra, KK, 705, 722

Kandampully, J, 867

Kanninen, B, 866

Kara, SM, 631, 638

Karagiannisa, G, 75, 84

Karamustafa, K, 153, 167, 202, 206 
Karmali, S, 294

Kaspar, C, 673, 679

Kastenholz, E, 550, 566, 567, 569

Katircioglu, ST, 632, 638

Kato, A, 10, 225, 255

Katsikeas, CS, 139, 142, 168

Kaynak, E, 150, 166

Kazi, S, 696, 702, 703

Keller, P, 206, 413, 603, 680, 681, 683, 702

Keller, WJ, 52, 64

Kellert, S, 384

Kennedy, G, 439

Kent, J, 841

Kenyon, W, 440

Keske, CM, 873, 883

Ketchen, DJ Jr, 95, 102

Khaled, M, 439

Khan, H, 7, 105, 106, 118

Khan, M, 43, 63

Khanna, DK, 166

Kierzkowski, H, 577, 579, 602

Kiesecker, J, 847, 866

Kikuchi, T, 26, 27, 885, 910

Kildow, J, 364, 387

Kim, E, 490

Kim, HJ, 631, 638

Kim, K, 473, 480, 486, 490

Kim, Y-S, 548, 569

Kindleberger, CP, 62, 140, 166

King, B, 315

King, OH, 370, 386

King, S, 439

Kiolberg, J, 809

Kirkpatrick, S, 466

Kirsner, S, 260, 278

Klaassen, L, 413, 414

Klein, LR, 50, 59, 64

Klein, RA, 339, 346, 349, 353, 358

Klein, YL, 362, 386

Klenosky, DB, 733, 739

Klepper, S, 228, 252, 253

Klineberg, S, 854, 866

Knetsch, J, 260, 277

Knight, AT, 310, 938

Knowles, T, 137, 576, 784, 167, 602

Kobayashi, S, 892, 909

Kock, S, 149, 150, 152, 162

Kofana, GE, 752, 757

Kohler, W, 579, 602

Kolbert, E, 814, 815, 840
Konan, DE, 473, 480, 486, 490

Konrad, W, 291, 295

Kosaka, W, 908

Kotchen, MJ, 287, 295

Kotler, P, 100

Kralj, A, 867

Kreishan, FM, 631, 638

Krippendorf, J, 655, 679

Krueger, AO, 622, 625, 638

Krüger, O, 824, 825, 829, 834, 840

Krugman, PA, 577, 602

Krugman, PR, 37, 64

Krutilla, JV, 873, 883

Krystallis, A, 847, 855, 866

Kucukusta, D, 295

Kuilamu, M, 751, 755, 758

Kuivalainen, O, 150, 167

Kula, E, 369, 386

Kulesa, P, 382, 385

Kumar, S, 284, 286, 288, 295, 811

Kundu, SK, 153-156, 158, 159, 163

Kunzig, R, 814-816, 840

Kuronuma, Y, 26, 27, 885, 910

Kurzweil, R, 817, 840

Kusluvan, S, 153, 167

Kuznets, SS, 227-230, 233, 237, 241, 246, 249, 253

Kwon, K, 545, 547, 548, 569

Kwortnik, RJ, 346, 349, 358

Kyläheiko, K, 167

Labonte, R, 295

La Croix, S, 249, 253

Lado-Cousté, N, 161

Laesser, C, 194, 206, 548, 569

Laffargue, J-P, 617

Lam, S, 862, 864

Lama, M, 22, 705, 713, 722, 723

Lam-Po-Tang, J, 287, 295

Lancaster, G, 199, 206

Lancaster, KJ, 211, 222, 369, 386

Landau, S, 102

Landgrave, R, 387

Lane, B, 794, 798, 808

Laney, LO, 524, 525, 540

Langford, I, 440

Langford, IH, 364, 384

Lansdell, N, 409, 413

Lanza, A, 73, 84, 623, 624, 637, 639, 640

Lanzilotta, B, 637 
Laroche, M, 863, 866

Dwyer, L, vii, 18, 521

Lau, L, 57, 59, 60, 62, 64, 67, 84

Laurance, WF, 815, 840

Law, R, 34, 62, 194, 195, 205

Lawson, AM, 540

Lawton, C, 222

Layard, R, 413

Lazarevski, K, 100, 102

Lazarow, N, 13, 14, 16, 361, 363, 371, 374, 377, 386-388, 443, 447, 448, 452, 456, 457, 464, 466, 467

Leakey, R, 815, 831, 841

Leamer, E, 384

Leamer, EE, 43, 64

Lean, HH, 631, 632, 639

Leask, A, 259, 278, 279, 337

Leakey, R, 823

Ledesma-Rodríguez, F, 639

Lee, C, 94, 103, 545, 547, 548

Lee, C-K, 474, 482, 486, 491, 547, 569

Lee, CC, 634, 639

Lee, D, 439, 473, 484, 486, 492

Lee, HC, 115, 118

Lee, M, 601

Lee, YW, 57, 61

Leese, M, 102

Leeuwen, ES, 548, 569

Leeworthy, VR, 371, 386

Legohérel, P, 548, 570

Lei, Z, 219

Leiper, N, 685, 703

Leisch, F, 92, 96, 98, 101, 102, 104

Lejárraga, I, 545, 570

Lemoine, F, 578, 602

Lennon, J, 262, 271, 277, 278

Lennon, JJ, 489, 492

Léon, CJ, 627, 639

Leones, J, 545, 548, 565, 570

Leontief, W, 53, 525, 540

Lester, J-A, 355, 358

Leujak, W, 929, 932, 938

Leung, P, 18, 492, 572

Leung, PS, 523, 541

Levy, A, 867

Lewbel, 53, 75

Lewbell, A, 75, 84

Lewin, R, 841

Leyshon, A, 163

Li, G, 65, 68, 69, 75, 84, 85, 118
Li, J, 152, 167

Li, S, 152, 473, 478, 482-485, 491

Liberto, 637

Libreros, M, 498, 520

Lichty, RW, 545, 570

Liebhold, D, 840

Lim, C, 34, 64

Lima, J, 548, 570

Limburg, KE, 385, 915, 937, 938

Lime, DW, 805, 809

Lindberg, K, 26, 29, 435, 439, 874, 883

Lindqvist, M, 143, 167

Lindsay, V, 161

Linnemann, H, 143, 167

Lins, RL, 295

Linsheng Zhong, JD, 726, 727, 739

Lionetti, S, 637

Lipsey, RE, 177, 189

Litteljohn, D, 138, 153, 158, 167

Liu, 185, 189, 388, 545, 547, 548, 552, 565,570

Lockwood, 153, 154, 161, 278, 369, 386

Lockyer, T, 413

Loke, MK, 492

Loker-Murphy, L, 735, 739

Lolohea, S, 746, 749, 758

London, GM, 284, 296

Long, D, 892, 909

Loomis, J, 377, 383, 386, 387, 434, 440, $866,873,883$

Lordan, G, vi, 12, 281, 297

Louviere, 384

Lovelock, CH, 151, 167

Lovett, J, 434, 441

Lowes, B, 679

Lowrie, EG, 284, 296

Lowry, W, 434, 439

Lozano, J, 627, 638, 639

Lü, JY, 189

Lu, LJ, 617

Lucas, RC, 809, 816

Lucas, RE, 620, 624, 639

Lück, M, 358

Ludvigson, T, 758

Lumsdon, L, 566, 568

Lundberg, DE, 735, 739

Lundie, S, 490, 771, 783

Luostarinen, R, 138, 170

Lurong, C, 602 
Lütkepohl, H, 628, 629, 637, 639

Lyssiotou, P, 68, 69, 72, 73, 75, 84

Ma, X, 434, 440

MacDonald, GM, 228, 253

MacReady, N, 281, 285, 286, 296

Madau, FA, 783

Maddah, B, 353, 358

Madden, J, 472, 482, 484, 490, 491, 510,520

Maddison, D, 767, 784

Madsen, TK, 145, 167

Magee, SP, 43, 64

Mahajan, VAP, 694, 703

Mahon, BD, 283, 296

Mahoney, A, 548, 571

Mahoney, D, 937

Mahooney, J, 865

Maitra, AK, 445, 466

Mak, vi, vii, 10, 18, 179, 189, 225, 229,

233, 236, 237, 244, 252, 253, 255, 345, 358, 426, 430, 439, 440, 523, 541

Maltby, E, 940

Manaktola, K, 847, 866

Manente, M, 545

Manfredo, MJ, 385

Mangion, ML, 68, 69, 75, 84

Mankiw, NG, 621, 639

Manning, 752, 758, 939

Manuela, P, 62

Marin, G, 94, 103

Mark, J, 83

Markandya, A, 772, 784, 810

Market Trends Pacific and John M. Knox \& Associates, Inc., 244, 253

Markowitz, H, 140, 167

Markusen, JR, 188, 579, 602

Marriotti, A, 673, 679

Marsden, S, 445, 466

Marshall, 94, 103, 621, 639

Marti, BE, 339, 346, 358

Martin, BS, 229, 253

Martin, EA, 663, 679

Martin, F, 170

Martin, J, 294

Martin, KA-M, 150, 163

Martin, LJ, 284, 296

Martinetz, T, 97, 103

Martínez, ML, 362, 387

Martín-Morales, N, 638
Maskus, K, 188

Maslin, C, 865

Massieu, A, 520

Massy, WF, 102

Matečić, I, 205

Matheson, A, 412

Mathieson, A, 543-545, 547, 570, 647, 672, 679, 683-685, 703, 704, 805, 809

Mathieu, L, 434, 440

Mattsson, L, 145, 166

Matus, S, 864

Mauer, 147

Mayer, M, 548, 570

Mays, D, 297

Mazanec, J, 88, 90, 100, 103, 104

McAuley, A, 138, 145, 163

McCabe, S, 694, 696, 698, 703

McCahill, L, 937

McCalla, R, 343, 357

McCarthy, 355, 358, 805, 809

McCaughey, N, 491

McClung, G, 319, 330, 336

McDonald, JM, 385

McDonnell, I, 124, 132

McDougall, PP, 144, 145, 167, 168

McEniff, J, 327, 336

McFadden, DL, 61

McGehee, NG, 752, 758

McGregor, PG, 490

McIntosh, B, 937

McIntosh, RW, 206

McIntyre, G, 789, 809

McKay, D, 295, 451

McKeever, M, 866

McKenney, B, 847, 866

McKinnon, RI, 622, 639

McLaren, KR, 57, 59, 62

McLellan, RW, 84

McLennan, C, 880, 883

McMahon-Beattie, U, 492

McManus, JC, 147, 167

McNamara, A, 880, 883

McNeely, JA, 774, 784

McNeill, JR, 814, 841

McQueen, M, 153, 154, 158, 164, 181, 182, $189,578,601$

Medda, F, 357

Medical Tourism Association, 290, 296

Medina-Muñoz, DR, 202, 206

Medina-Muñoz, JM, 206 
Medio, D, 928, 932, 939

Medlik, S, 577, 601

Meis, S, 520

Melendy, HB, 237, 253

Mello, 68

Melo, C, 519, 846, 866

Mergos, GJ, 75, 84

Metzger, T, 297

Michalko, A, 868

Mihalič, T, 20, 21, 655, 660, 668, 674, 679, 681

Mike Raybould, 16, 361, 388, 443, 467

Mill, JS, 36, 64

Mill, RC, 685, 703

Millennium Ecosystem Assessment, 916, 939

Miller, G, 847, 866

Miller, GR, 808

Miller, ML, 363, 386, 387

Milne, S, 277, 744

Milner, C, 474, 480, 490

Minas, S, 838

Ministry of Environment, 886, 887, 889, 890, 893, 894, 909

Miossec, JM, 686, 703

Mishan, EJ, 395, 403, 413

Mishio, M, 909

Mishra, HR, 834, 841

Mitchell, J, 747, 758

Mitchell, RC, 368, 387

Mitra, A, 22, 705, 709, 713, 714, 718, 722,723

Mittermeier, CG, 841

Mittermeier, RA, 841

Mjelde, JW, 491

Moberg, F, 915, 916, 919, 920, 939

Moeltner, K, 385

Moeti, KB, 142, 167

Mohan, V, 606, 617

Mok, C, 548, 570

Mondher Sahli, 20, 575, 603, 619, 640

Montfort, KV, 568

Moon, S, 491

Moore, E, 874, 883

Moore, S, 123, 133

Moorthy, A, 387

Moosa, IA, 73, 83, 148

Moran, D, 872, 884

More, T, 434, 435, 440

Moreira, AC, 140, 146, 167
Morgan, RE, 139, 142, 168

Morgan, T, 249, 254

Morimoto, K, 908

Morley, CL, 34, 64, 211, 222

Morling, P, 938

Morris, D, 814, 841

Morrison, AM, 90, 102

Morrison, S, 238, 240, 241, 254

Morritt, R, 100, 103

Moscardo, G, 262, 278, 846, 866

Moschini, G, 76, 84

Moussawi-Haidar, L, 358

Movono, A, 751, 755, 758

Moyo, D, 822, 836, 837, 841

Mudambi, R, 153, 161, 548, 566, 570

Muellbauer, J, 44-46, 50, 54-56, 63

Mules, T, 394, 399, 407, 412, 413, 445, 466

Mullarkey, DJ, 384

Müller, FG, 774, 784

Müller, M, 570

Muller, M, 104

Munday, A, 500, 519

Munro, HJ, 138, 145, 163

Murray, T, 42, 64

Myers, JH, 88, 103

Myers, N, 820, 825, 841

Nabin, M, 617

NACS-J, 886, 887, 892, 898, 904, 906, 909

Naeem, S, 385, 937

Nahata, B, 330, 336

Naidu, V, 751, 759

Nakra, P, 285, 296

Narayan, PK, 473, 475, 482, 483, 491, $545,570,745,758$

Narayan, S, 758

Narayanasamy, N, 845, 867

Narsey, W, 745, 758

Nash, D, 652, 679

National Audit Office, 270, 278

National Commission on Macroeconomics and Health, 292, 296

Navaro, P, 124, 132

Neary, JP, 609, 617

Nelsen, C, 375, 377, 387

Nelson, CK, 628

Nelson, CR, 639

New South Wales Government, 365, 387 
New South Wales National Parks and Wildlife Service, 875, 883

Newman, RS, 693, 696, 703

Newsweek, 283, 296

$\mathrm{Ng}, \mathrm{S}, 75,84$

Ng, Y-K, 423, 439

NGM, 815, 841

Nguyen, D, 868

Niccolucci, V, 841

Nicholas, S 153, 156, 168

Nicholls, RJ, 362, 387

Nicholson, R, 729, 739

Nijkamp, P, 568-570

Nilsson, PA, 340, 344, 347, 358

Norbert Vanhove, 14, 393, 418

Norgaard, R, 384

Noriega, R, 466

Norman, R, 199, 206

Norman, V, 37, 63

Norman, WC, 568

Noronha, F, 696, 703

Noronha, L, 698, 703

North, DC, 639

Norton, B, 384

Norton, LP, 281, 286, 296

Novelli, M, 648, 678

Nowak, J-J, 579, 585, 587, 602, 623, 625, $635,637-639,662,679$

Noyes, C, 868

Nsiah, C, 638

NSSO, 125, 127, 131, 132

NSW Department of Infrastructure,

Planning and Natural Resources, 771, 784

NTA (National Tourism Administration of China), 726, 739

Nunes, PM, 633, 640

Nunez-Lara, E, 782

Nyaupane, G, 260, 278

Nystrom, H, 164

Nyström, M, 919, 920, 939

O'Brien, D, 232, 237, 254

O'Connell, JF, 223

O'Connor, S, 775, 784

O'Farrell, PN, 150, 152, 168

O'Hagan, JW, 41-43, 68, 69, 64, 84

O'Neill, JW, 190

O'Neill, RV, 385, 937, 938

Oates, WE, 778, 782, 783
Obermiller, F, 569

Obstfeld, M, 37, 64, 602

OECD Education at a Glance, 315

Office of Economic and Statistical

Research, 880, 883

Office of Travel and Tourism

Industries, 248

Ogasawara Ecotourism Congress, 898, 909

Ogasawara Tourist Association, 893, 909

Ogasawra Tourist Association, 893

Ogilvie, FW, 545, 570

Oh, C, 631, 639

Ohlin, B, 37, 168, 593, 676

Oka, S, 902-904, 909

Oka Shuichi, 901, 905

Okotai, P, 370, 385

Oliver, D, 326, 336

Olsen, MD, 150, 156, 170

OmniTrak, Inc., 244, 254

Ono, M, 892, 909

Oppermann, M, 735, 739

Organisation for Economic Co-operation and Development (OECD), 12, 35, 84, 194, 206, 291, 300, 301, 303, 315, 497, 501, 519, 520, 540, 575, 578, 590, 633, 639, 775, 781, 784, 910, 940

Ormiston, D, 933, 939

Ormond, RFG, 939

Ornelas, E, 581, 602

Orru, E, 783

Ortúzar, JDD, 925, 939

Osborne, LL, 386

Osleeb, JP, 386

Ostaszewski, K, 336

Ostrom, E, 27, 29, 872, 883

Oum, TH, 238, 254

Oviatt, BM, 144, 145, 167, 168

Owen, CB, 547, 568

Ozanne, L, 868

Ozuna, T Jr, 569

Paavola, J, 940

Pacific Asia Travel Association, 118

Pack, A, 83

Packer, C, 295

Padachi, K, 640

Pagan, JA, 295

Page, S, 208, 222

Page, SJ, 570

Palaskas, T, 216, 222 
Palmquist, RB, 387

Pambudi, D, 474, 477, 491, 520, 875, 883

Pandit, S, 864

Panella, G, 767, 769, 770, 783, 784

Panoho, J, 758

Panthera, 821, 841

Panvisavas, V, 154, 168

Panzar, J, 277

Papadopoulos, S, 153, 162

Papathanassis, A, 341, 358

Papatheodorou, A, 9, 10, 29, 68, 69, 84, 207, 208, 211, 212, 214, 218, 219, 222, 223, 278, 345, 358, 413, 680

Parameshwaran, P, 62

Parikh, K, 126, 132

Park, DB, 739

Parks and Wildlife Service Tasmania, 440

Parmenter, 472-475

Parmenter, B, 519

Parmenter, BR, 488, 489

Parry, R, 937

Parsons, G, 434, 440

Parsons, W, 123, 132

Paruelo, J, 385, 937

Pashardes, P, 57, 64

Pasquale M Sgro, 605, 618

Pass, C, 654, 679

Pass, CL, 162

Patterson, PG, 150, 151, 168, 170

Patterson, TM, 830, 841

Paulhus, DL, 94, 103

Payne, J, 384

Pearce, 545, 729

Pearce, D, 265, 278, 412, 652, 658, 679, 739, 884

Pearce, DG, 545, 570, 729, 739

Pearce, DW, 363, 387, 412, 413, 790, 802, 810, 872

Pearson, M, 939

Pearson, MP, 913, 939

Peel, V, 750, 758

Peerapatdit, N, 571

Pegg, S, 882, 883

Pelat, C, 287, 296

Pendleton, L, 364, 387

Pennings, G, 284, 296

Perdue, R, 735, 739, 758

Pereira, SC, 189

Pereyra, JS, 637

Perez, EA, 548, 571
Pérez-Maqueo, O, 387

Pérez-Rodríguez, JV, 639

PERSGA, 928, 932, 939

Personal Property Advisor, 676, 679

Peters, M, 104, 150, 153, 169, 647, 661, 679

Peterson, GL, 385

Peterson, ME, 810

Petit, S, 19, 582, 602, 679

Petrick, J, 347, 357, 358

Petros Zenelis, 207, 224

Pezzey, J, 789, 810

Pham, DT, 500, 510, 511, 520, 570

Pham, T, 18, 520, 875, 884

Pham, TD, 493, 520

Phang, SY, 118

Phillips, O, 274, 278, 330, 336

Phillips, P, 161, 177, 189, 746, 750, 758

Phlips, L, 44, 48, 50, 58, 64

Piggot, TL, 445, 447, 466

Pigliaru, F, 623, 624, 637, 639

Pigou, AC, 417, 420, 438, 440, 546, 803,810

Pinazzo, J, 440

Pinder, D, 868

Pine, R, 177, 178, 181, 186, 189

Ping Yin, Y, 490

Pitts, WD, 908

Pizam, A, 548, 571

Pla Barber, J, 150, 153, 155, 157, 159, 168, 169

Planet Green, 830, 841

Planin, J, 679

Planina, J, 668, 673, 674

Planting, 540

Plomp, J, 283, 296

Plosser, CI, 628, 639

Pohlmann, C, 277

Pointeau, R, 466

Pollak, RA, 52, 53, 65, 74, 84

Pollard, 283, 296

Polman, L, 836, 841

Polo, C, 473, 476, 491

Polunin, I, 668, 680

Pomeroy, RR, 571

Poortinga, YH, 103

Porter, PK, 548, 571

Portney, P, 384

Posner, R, 418, 440

Poterba, JM, 61 
Powell, AA, 44, 49, 51, 52, 65

Prasad, A, 758

Prasad, BC, 22, 23, 745, 750, 758, 761

Pratt, S, 18, 473, 485-487, 491, 492, 744,757

Prescott, K, 162

Prest, A, 394, 413

Price Waterhouse Coopers (PWC), 466

Price, L, 162

Prideaux, B, 849, 867

Priestley, G, 278

Primont, D, 62

Proença, S, 633, 639

Pruy, A, 297

Pulina, M, 83, 632, 637

Pulina, $\mathrm{P}, 783$

Purcell, W, 153, 156, 168

Puumalainen, K, 167

Pyo, SS, 68, 71, 84

Qi, PS, 178, 189

Quandt, RE, 16, 29, 212, 223

Quaschning, T, 297

Queensland Government, 445, 466

Quer, D, 155, 158, 168

Qureshy, L, 703

Radam, A, 785

Radtke, H, 569

Raguraman, K, 547, 548, 571

Raicola, V, 749, 758

Rainforest Alliance, 828, 841

Rajaratnam, D, 151, 162

Ramaswami, SN, 140, 160

Ramirez, R, 199, 206

Ramón Rodríguez, A, 150, 153-156, 158, 159, 168

Ramos, M, 640

Ramsey, FP, 420, 440, 620, 639

Randall, A, 381, 386

Randner, R, 384

Rao, CP, 149, 150, 152, 165

Raskin, RG, 385, 937

Ravilious, C, 939

Rawls, J, 444, 466

Raybould, M, 13, 14, 363, 364, 387, 413, 445, 447, 448, 452, 456, 457, 466

R Development Core Team, 96, 103

Redekop, WK, 296

Reed, B, 937
Reed, CM, 285, 286, 289, 290, 296

Reichel, A, 548, 571

Reimer, JJ, 487, 491

Reinhardt, F, 847, 867

Reiseanalyse, RA, 195, 206

Reserve Bank of Australia (RBA), 300, 315

Resiman, D, 282, 296

Reyers, B, 938

Rey-Maquieira, 638-640

Rialp, A, 146, 168

Rialp, J, 146, 168

Richards, G, 321, 336

Richards, JF, 814, 841

Richards, S, 270, 278

Richardson, R, 434, 440

Rietveld, P, 569

Rigall-I-Torrent, R, 872, 884

Riley, R, 275, 278

Rimmer, M, 506, 519

Risso, 637, 640

Ritchie, J, 674, 680

Ritchie, JR, 727, 739

Ritchie, JRB, 206

Rizvi, SAT, 937

Rizzo, DM 937

Roberts, A, 519

Roberts, J, 150, 168

Roberts, CM, 929, 931, 935, 938

Robertson, M, 118

Robilliard, A-S, 487, 490

Robinson, E, 141, 168

Robinson, S, 519

Roe, B, 867

Rogers, H, 266, 278

Rogers, R, 103

Rojid, S, 640

Romano-Critchley, G, 294

Rome, A, 846, 866

Romer, D, 622, 639

Romer, PM, 620, 639

Rompf, P, 571

Root, FR, 148

Roper, A, 162, 167

Rosalsky, MC, 63

Rose, E, 161

Rose, JM, 938

Rosenberg, N, 254

Rosenthal, LH, 384

Rosenzweig, JA, 286, 295 
Rossello, J, 223

Rossi-Hansberg, E, 577, 579, 582, 601

Roster, CA, 94, 103

Rothenbach, B, 866

Rouget, M, 938

Round, D, 305, 315

Routledge, P, 696, 703

Rowe, T, 847, 867

Roxburgh, T, 918, 939

Roy, J, 345, 351, 358

Roy, KC, 703, 722, 770, 784

Royal Automobile Club of Queensland, 466

Rubin, H, 50, 59, 64

Rugg, D, 211, 223

Rugman, A, 141, 147, 161, 169

Ruitenbeek, HJ, 921, 939

Runnels, V, 295

Russel, RR, 62

Russell, C, 384

Russell, M, 751, 752, 759

Russo, A, 278

Rutherford, T, 506, 519

Ryan, C, 76, 177, 189, 403, 413, 433, 440, 756,759

Ryan, DL, 76, 84

Saarenketo, S, 167

Sabadani, B, 295

Sacks, J, 172

Sacramento, I, 150, 169

Sahli, M, 19, 579, 602, 637-639, 679

Sahoo, P, 336

Sakai, MY, 68, 70, 84

Sallis, J, 162

Salm, RV, 928, 939

Salma, U, 499, 505, 520

Samartín-Sáenz, M, 161

Samoan Bureau of Statistics, 749, 759

Samoan Tourism Authority, 749, 759

Sampol, CJ, 548, 571

Samuelson, PA, 44, 65, 139, 169, 582, 601,602

Samy, J, 750, 759

Sánchez Peinado, E, 150, 169

Sanchez, M, 866

Sandford, D, 665, 680

Santana-Gallego, M, 634, 639

Sanz, J, 277

Sargan, JD, 111, 118
Sassone, PG, 403, 413

Sathiendrakumar, R, 787, 788, 790, 794, 801, 803, 810

Savin, NE, 62, 74, 82

Saving Rhinos, 823, 841

Sawamiphakdi, D, 545, 571

Scaperlanda, A, 43, 61, 68, 69, 82, 147

Scarfe, B, 377, 387

Schaffer, WA, 403, 413

Scherly, F, 402, 413

Scheurer, R, 326, 337

Scheyvens, R, 751, 752, 755, 759

Schlegelmilch, B, 865

Schmidt, J, 291, 294

Schmitt, RC, 233, 237, 240, 243, 249, 251, 254

Schneider, L, 847, 867

Schott, C, 848, 857, 867

Schubert, F, 623, 625, 867

Schubert, SF, 640, 854

Schulten, K, 97, 103

Schultz, S, 434, 435, 440

Schultz, TW, 621, 640

Schuman, H, 383, 384, 387

Schumpeter, JS, 11, 29, 264, 278

Schuster, G, 836, 841

Schved, JF, 295

Scott, N, 882, 883

Scura, L, 938

Seabrooke, W, 385

SEAM, 926, 927, 939

Sebastiáan, M, 150, 169

Seetanah, B, 565, 571, 640, 634

Seifert-Granzin, J, 698, 703

Sen, AK, 125, 132

Sen, G, 694, 696, 698, 703

Sengupta, A, 133

Sequeria, TN, 633, 640

Seraj, K, 857, 867

Servais, P, 145, 167

Severt, D, 571

Seymour, M, 123, 133

Sgro, M, 617

Sgro, PM, vii, 606, 617, 623, 638

Sgro, PS, 617

Shanahan, M, 305, 315

Shane, S, 167

Sharma AM, 294

Sharma, DD, 162

Sharma, G, 864 
Sharpley, R, 645-647, 652, 668, 679, 680

Shaw, G, 452, 664, 668, 681

Sheehey, C, 358

Shehata, AE, 913, 939

Shelby, B, 932, 939

Sheldon, PJ, 34, 65

Shelton, D, 937

Sheng, J, 308, 315

Shephard, R, 45, 65

Sheppard, 95, 103

Shimakawa, T, 889, 909

Shimizu, Y, 891, 892, 909

Shook, CL, 95, 102

Short, AD, 460, 466

Shoven, JB, 485, 491, 506, 520

Shrader, RC, 167

Shuib, A, 785

Shumiya, T, 895, 903, 908

Sigala, M, 220, 223

Sillano, M, 925, 939

Silva, MSM, 169

Simcock, N, 866

Simkin, L, 90, 101

Simmons, DG, 520, 865

Simon, H, 6, 29

Simpatico, T, 937

Sinclair, M, 278

Sinclair, MT, 68, 69, 75, 84, 223, 472, 474, 476-478, 480, 490, 492, 638, 680

Sinclair, T, 29, 413, 578, 585, 602, 610, 617, 789, 791, 808

Singapore Tourism Board, 106, 118, 119

Singer, P, 817, 841

Singer, S, 294

Sinha, C, 726, 740

Sinkovics, R, 865

Siqueira, A, 696, 702, 703

Sivakumar, K, 149, 150, 164

Six Flags, 321, 323-325, 327, 331, 332, $334,335,337,677$

Sjöholm, F, 177, 189

Skene, J, 472, 491, 492, 770, 784

Slatter, C, 752, 759

Slaughter, MJ, 189

Small, C, 387

Smeral, E, 70, 524, 525, 527, 540, 575, $603,664,676,680$

Smidt, S, 412

Smith, A, 65, 621, 640

Smith, AW, 445, 466
Smith, FT, 315

Smith, KR, 254

Smith, M, 295

Smith, P, 294

Smith, S, 209, 413

Smith, SC, 652, 653, 680

Smith, SLJ, 770, 784

Smith, V, 262, 278, 679

Smith, VK, 369, 386, 387

Smith, VL, 686, 703

Smith, W, 87, 103

Smits, W, 841

Smyth, R, 491

Snapp, R, 937

Söderqvist, T, 940

Sofer, M, 751, 759

Sofield, T, 751, 759

Solnet, D, 867

Solow, RM, 61, 384, 620, 640

Sommerville, A, 294

Song, H, 34, 65, 85, 111, 118, 166, 295

Soriani, S, 343, 350, 358

Soskin, M, 264, 277

Soukiazis, E, 633, 639

South Pacific Tourism Organisation, 747, 750,759

Spalding, MD, 920, 939

Spash, CL, 368, 386

Spencer, A, 124, 132

Spotts, DM, 548, 571

Spurgeon, J, 918, 939

Spurr, R, 412, 490, 500, 503, 504, 520, $568,783,883,884$

Spurr, T, 412

Spurrm, R, 519

Sreekesh, S, 703

SSRDP, 926, 927, 940

Stabler, M, 15, 211, 219, 220, 272, 278, 395, 396, 402, 404, 406, 411-413, 578, $585,602,658,680,847,867$

Stankey, GH, 805, 806, 809, 810

Stanton, PJ, 65

Stare, M, 150, 169

State of Hawaii, Department of Business, Economic Development and Tourism, 240, 246, 248, 251, 253, 254

Statistics Canada, 670, 671, 680

Statistics New Zealand, 670, 680

Steel, F, 340, 358

Steenge, AE, 545, 571 
Steines, DN, 545, 547, 570

Stern, RM, 43, 64

Stevens, T, 434, 435, 440

Stiglitz, J, 431-433, 790, 440, 810

St. John-Ives, E, 751, 759

Stocks, J, 694, 696, 698, 703

Stoeckl, N, 25, 845, 847, 867, 869

Stoklossa, CJ, 294

Stone, EA, 386

Stone, JRN, 60, 65

Štrach, P, 150, 165

Strauss, D, 466

Strout, AM, 622, 637

Stuart, G, 466

Sugden, R, 395, 399, 166, 413

Sugiyarto, G, 473, 478, 485, 489, 492

Sultan, E, 547, 569

Sunlu, U, 828, 842

Sunshine Coast Regional Council (SCRC), 447, 460, 466

Surfing Australia, 371, 375, 388

Sustainable Tourism Development Consortium, 750-752, 759

Suter, G, 384

Sutherland, W, 283, 294

Sutton, B, 519

Sutton, P, 385, 387, 937

Swait, J, 384

Swales, JK, 490

Swan, TW, 620, 621, 640

Swedenborg, B, 147, 169

Sweeting, J, 864

Swethurst, DP, 283, 296

Sydney Morning Herald, 434, 440

Sylvain Petit, 575, 603

Tabatchnaia-Tamirisa, N, 481, 492

Tadasse, B, 638

Takahashi, S, 94, 104

Tamasese, E, 751, 759

Tamberi, M, 588, 601

Tan, F, 7, 105, 119

Tang, CF, 631, 632, 639

Tang, FF, 179, 190

Tata Economic Consultancy Services, 125, 133, 703

Tauber, E, 88, 103

Taufatofua, RG, 749, 759

Tawfik, RT, viii, 27, 425, 911, 923, 940

Taylor, JS, 154, 168
Taylor, S, 156, 169

Taylor, T, 547, 569

TDA, 911, 926, 940

TECS, 691, 695, 696, 703

Tefft, N, 287, 296

Teisl, M, 847, 867

Teles, V, 489, 678

Telfer, DJ, 645, 647, 668, 679, 680

Terasvirta, T, 76, 83

TERI, 696, 703

Terpstra, V, 150, 169

Thaler, RH, 6

Thapa, P, 472, 491

Thapa, PJ, 510, 520

The Australian, 307, 309, 310, 312, 314, 315, 388

The China Business Review, 182, 190

The Daily Telegraph, 434, 440

The Economist, 312, 315

The Gallup Organization, 205

The Local, 314, 315

Theil, H, 44, 51, 52, 58, 59, 65

Thomas Cook, 193, 200, 201, 206

Thomas, B, 439, 693, 702

Thomas, RL, 42, 46, 48, 53, 56, 59, 65

Thomas, SE, 283, 297

Thompson, D, 57, 808, 852, 860, 867

Thrift, N, 239, 163

Thur, S, 434, 440

Tian, P, 18, 359

Tian, Y, 185, 189

Tietenberg, J, 802, 804, 810

Tigers in Crisis, 827, 841

Timothy, D, 260, 278

Timur, A, 570

Tirole, J, 581, 603

Tisdell, CA, 6, 14-16, 20, 21, 24, 26, 28, $30,37,65,88,101,115,118,124-126$, 133, 173, 190, 229, 248, 249, 254, 401, 418, 420, 421, 425, 431-436, 440, 441, 459, 463, 606, 617, 645-647, 680, 705, $722,734,745,769,770,775-778,780$, $784,788,794,799,804,808,810,819$, $828,829,831,832,834,841,842,928$, 930,931

Tivy, J, 805, 810

Tiwari, AK, 633, 640

Tjolle, V, 847, 867

Toda, HY, 628, 640

Todaro, MP, 611, 617, 652, 653, 680 
Toh, RS, 118

Tomlinson, R, 466

Tooke, N, 275, 278

Toriki, S, 358

Tosi, J, 840

Tourism Council of the South Pacific (TCSP), 747, 759

Tourism Research Australia, 875, 876, 880,884

Toyoda, T, 897, 900, 901, 909

Travel Journal, 246, 247, 254

Triandis, HC, 94, 102

Tribe, A, 769, 783

Tribe, J, 645, 647, 680

Triffin, R, 4, 30

TRIP Consultants, 746, 759

Trujillo, L, 357

Tsai, P, 140, 169

Tsampra, M, 222

TSAs, 19

Tucker, KA, 492

TUI, 195, 199-203, 206, 214

Turbelin, C, 296

Turgut, V, 570

Turner, D, 809

Turner, JL, 363, 364, 581, 602

Turner, RK, 387, 388, 425, 490, 790, 914-916

Turner, L, 293, 297

Turner, RK, 27, 465, 810, 938, 940

Turner, T, 837

Turvey, R, 394, 413

Tversky, A, 6

Ucbasaran, D, 170

Ullal, J, 841

Um, S, 569

UN, 119, 497, 519, 524, 541, 578, 580, 603, 648-652, 680, 836, 837

Ünal-Kesenci, D, 578, 602

UNCTC, 154, 169

UNDESA, 650-652, 667, 680

UNDP, 647, 648, 680, 692, 704, 836

UNEP, 828, 842, 846, 867, 921, 939, 940

UNESC, 152, 154, 169

UNESCAP, 742, 744, 746, 759, 760, 776, 780,785

UNESCO, 126, 130, 299, 300, 316, 716, $726,758,820,907,909$
United Nations, 35, 65, 154, 413, 525, 540, 604, 640, 692, 909, 921

United Nations and World Tourism Organization, 540

United Nations Conference on Trade and Development, 603

United Nations Statistics Division (UNSD), 520, 540

United Nations World Tourism

Organisation (UNWTO), 497, 517, 520, $521,603,680,867$

University of Queensland, x, 3, 30, 281, 299, 306, 314, 316, 417, 441, 465, 493, $520,761,784,785,871$

Untong, AK, 632, 640

UNWTO (United Nations World Tourism Organisation), 183, 198, 206-209, 229, 496, 526, 529, 540, 576, 646, 648-652, 666, 667, 669, 680, 681, 682, 692, 703, $743,745,760,766,784,788,811,817$, $846,867,868$

US Census Bureau, 240, 254

US Department of Commerce, Bureau of the Census, 255

US Department of Commerce, Office of Travel \& Tourism Industries, 255

US Government, Federal Inter-Agency River Basin Committee, Subcommittee on Benefits and Costs, 403, 413

USTOA, 192, 206

Uysal, M, 229, 253, 545, 571, 732, 739, 740

Uzawa, H, 49, 63

Vad, T, 150, 166

Vahlne, J-E, 142-145, 147, 166

Vaio, C, 345, 355, 357

Valente, S, 623, 624, 640

Valle, E, 473, 476, 491

Valleron, A-J, 296

Van Bueren, M, 370, 388

Van De Steeg, AM, 545, 571

van den Belt, M, 937

van den Bergh, JCJM, 940

van der Straaten, J, 940

Van Doren, C, 275, 278

van Heerden, JH, 482, 483, 489

van Herk, H, 94, 103

Van Ho, T, 490, 883

Van Ierland, EC, 938, 940

van Jaarsveld, AS, 938 
Van Koppen, K, 938

van Rekom, J, 262, 278

van't Hof, T, 938

Vanetti, M, 153, 166

Vanhove, N, 15, 394-396, 406, 413, 414, $647,663,664,668,672,681$

Vanuatu Tourism Development Office (VTDO), 748, 760

Var, T, 34, 545, 547, 548, 65, 570

Varian, H, 287, 294

Vaske, J, 854, 868

Vaughn, R, 387

Vázquez, G, 387

Vecina, FA, 295

Vellas, F, 677, 681

Verhallen, TMM, 103

Vermeer, D, 862, 868

Vernon, J, 847, 868

Vernon, R, 140, 147, 169

Veronneau, S, 345, 358

Vincent, D, 519

Vining, A, 412

Viola, MR, 386

Viu, J, 270, 279

Vivanco, L, 828, 842

Vlosky, R, 847, 851, 860, 864, 868

Voase, R, 260, 279

Vogel, N, 849, 868

Vos, R, 487, 492

$\mathrm{Vu}, \mathrm{L}, 294$

Wagar, JA, 805, 810

Wagner, JE, 545, 571

Wagner, TH, 294

Wahab, S, 413, 576, 603

Wahidin, K, 785

Wales, TJ, 52, 53, 59, 63, 65, 74, 76

Walkenhorts, P, 570

Walker, KE, 445, 466

Wall, G, 543-545, 547, 570, 571, 647, 672, 679, 683-685, 703, 704, 805, 809

Wallace, K, 914, 916, 940

Wallenstål, M, 152, 164

Walsh, C, 750, 760

Walters II, WG, 254

Walters, C, 301, 307, 311, 313, 316

Walters, D, 199, 206

Walton, GM, 252

Wang, HY, 190

Wang, R, 190
Wang, Y, 548, 571

Wanhill, S, 12, 13, 205, 230, 259, 261, 268, $278,279,317,318,320,321,327,337$, 429, 441, 568

Wanner, C, 284, 297

Ward, FA, 462, 466

Warford, JJ, 790, 802, 810

Warnken, J, 848, 868

Watson, A, 808

Watson, AE, 568

Wattanakamolchai, S, 758

Wattanakuljarus, A, 474, 480, 492, 665,681

Waugh, G, 775, 783

Waugh, RE, 545, 571

WCED, 790

Weaver, A, 342, 346, 349, 358

Weaver, DB, 818, 842

Weaver, JB, 287, 297

Weaver, SS, 297

Webber, AG, 663, 681

Wedel, M, 97, 100, 103

Weeden, C, 355, 358, 862, 866, 868

WEF, 217, 666, 681

Wei, SJ, 577, 600

Weidenfeld, A, 259, 279

Weiermair, K, 104, 150, 153, 169, 337

Weil, DN, 639

Weimer, D, 412

Weingessel, A, 101

Weinstein, AK, 150, 169

Weisfelder, CJ, 140-142, 169

Welch, LS, 138, 170

Welkenhuysen-Gybels, J, 94, 104

Wells, LT, 147, 170

Wells, MP, 819, 842

Welz, A, 938

Wen, J, 22, 126, 133, 173, 190, 432, 441, $725,726,734,740,799,810,819,841$

WES, 402, 412, 414, 415

West Gippsland Catchment Management Authority (WGCMA), 466

West, GR, 571

Westhead, P, 150, 170

Weston, R, 427, 441

Wet Tropics Management Authority, 849, 865,868

Whalley, J, 485, 491, 506, 520

Wheeler, DC, 284, 297

Whelan, T, 805, 810 
White, DS, 166

White, G, 458, 466

White, JT, 147, 161

White, KJ, 42, 65, 72, 73, 85

White, P, 434, 441

Whitehead, J, 853, 868

Whitton, T, 760

Wickens, MR, 112, 118

Wie, B-W, 345, 347, 359

Wiedersheim-Paul, F, 142, 166

Wight, PA, 818, 842

Wildlife Service Tasmania, 435

Wilkes, K, 270, 278

Wilkins, H, 315

Williams, A, 363, 388, 395, 399, 413, 664, 668,681

Williams, CL, 623, 628, 638

Williams, D, 868

Williams, HC, 283, 296

Williams, M, 384, 833, 842

Williamson, OE, 141, 170

Willig, R, 277

Wilson, C, 14-16, 26, 28, 30, 417, 432, 436, 441, 442, 780, 784

Wilson, D, 696, 704

Wilson, EO, 815, 816, 820, 828, 834

Wilson, JG, 388

Wilson, MA, 367, 388

Wind, Y, 102

Winsted, KF, 150, 170

Winston, C, 238, 240, 241, 254

Witt, CA, 109, 110, 663, 668, 681

Witt, SF, 65, 109, 110, 118, 663, 668, 679-681

Wittmer, A, 210, 221

Wolak, R, 149, 170

Wolf, S, 846, 866

Woltering, M, 570

Wong, K, 321, 337

Wong, KC, 118

Wong, KKF, 548, 570

Woo, S, 125, 133

Woo, WT, 172, 189

Wood, D, 874, 881, 882

Wood, EH, 545, 571

Wood, PA, 150, 152, 168

Wood, TJ, 283, 297

Woodroffe, CD, 366, 384
World Bank, 106, 118, 119, 133, 633, 646, 658, 681, 758, 781, 810, 836, $837,842,940$

World Commission on Environment and Development (WCED), 24, 790, 811

World Economic Forum, 681

World Tourism Organization (UNWTO), $229,520,523,540,617$

World Tourism Organization/United Nations, 601

World Trade Organization (WTO), 603

World Travel and Tourism Council

(WTTC), 497, 745, 760, 785

WRI, 815, 842

Wright, M, 170

Wright, R, 168, 814, 842

$\mathrm{Wu}, \mathrm{DC}, 70,732,85$

Xi, YP, 190

Xia, W, 852, 855, 868

Xiao, H, 181

Xiaodong, S, 353, 359

Yacob, MR, 774, 785

Yamamoto, T, 628, 640

Yanagida, JF, 492, 572

Yasui, T, 909

Yeoman, I, 474, 481, 489, 492

Yi, D, 291, 297

Yi, KM, 579, 601, 603

Yip, GS, 151, 167

Yochum, GR, 548, 567

Yong, J, 254

Yoo, JH, 101

Yoon, YS, 735, 739

Yoshida, K, 888, 895, 898, 909, 910

Yoshihara, K, 50, 51, 59, 65

Young, R, 447, 775, 785

Yu, C-M, 150, 169

$\mathrm{Yu}, \mathrm{ESH}, 617$

Yu, L, 8, 177, 179, 190

Yuan, M, 551, 571

Zachee, P, 295

Zakaria, F, 124, 133

Zapata, S, 355, 357

Zax, M, 94, 104

Zebregs, H, 696, 704

Zenelis, P, 9, 10, 216, 217

Zeng, Y, 852, 855, 868 
Zhang, CZ, 22, 725, 726, 740

Zhang, HQ, 189

Zhang, J, 473, 484, 486, 492

Zhang, K, 189

Zhang, X, 387

Zhangjiajie Statistical Office, 725 , 727,740

Zhao, JL, 150, 156, 170

Zheng, J, 168
Zhou, D, 472, 473, 475, 476, 486, 492, 548,572

Zientara, P, 752, 757

Zimmerman, F, 664, 681

Zimmermann, J, 284, 297

Zimmermann, KF, 287, 294

Zins, AH, 90, 94, 104

Zukin, S, 318, 321, 337 


\section{SUBJECT INDEX*}

access, 13, 16, 41, 129, 147, 180, 186-188, 193, 200, 213, 217, 221, 226, 260, 261, 264, 268, 285, 288, 289, 292, 293, 314, $321,326,331,344,362,363,365,370$, 383, 430, 431, 433, 446, 451, 459, 462, $622,655,669,676,697,701,735,749$, $769,777,819,835,883,889,899,908$, 917, 930, 931, 933

admission charges, 11, 259, 266, 272, 333 advanced economies, 648, 651, 652, 659,667

adventure tourism, 5, 711, 720

advertisement of tourism, government subsidies, 430

Afghanistan, 131, 207, 245

Africa, 324, 474, 591, 592, 594, 597, $632,634,636,641,678,760,775$, $822,826,865$

African countries, 132, 633, 826

African game reserves, 775

age of loneliness, 814,815

agencies, 192

agreement on guidelines, 891

agricultural, 4, 29, 63, 64, 83, 121, 130, 172, 269, 387, 388, 442, 478, 492, 541, 581, 584, 587, 666, 672, 751, 754, 759, $775,785,843,866-868,874,875,882$, 883,910

agricultural development, 769

aid trap, 836

AIDS, 55, 56, 59, 60, 64, 69, 72, 82, 84

AIDS demand system, 33, 52, 54, 56, 60, $62-64,67,68,73,83,84$

AIDS model, 57, 68, 69, 72, 82

AIDS model, non-linear, 57, 70, 71

Air Arabia, 216

Air Asia, 216

air cargo services, 213 air transport, 8-10, 207-209, 211,

213-215, 217-221, 497, 516, 586, 596

air transport accessibility, 216

air transport and tourism, 207, 209-211,

$217,221,224$

air transport sector, 209, 210, 221, 585

air transportation, 225, 233, 234, 236,

$238,242,243,248,249,251,345,532$, 537,538

air transportation services, 237, 238

airfares and flight times, 239

airline companies, 194, 196, 218, 219

airline sector, 9, 10, 213, 215, 586

airlines, 9, 10, 43, 106, 124, 152, 192, 196, 213-221, 223, 236-238, 240, 241, 245,

$353,433,439,477,529,537,580,734$

airport, 106, 207, 210, 218-221, 433, 475, 485, 676, 699, 709, 712, 727, 736,

737,747

airports as gateways to tourism destinations, 218

airports, importance for the local economies, 219

Alaska, 343

almost ideal demand system (see $A I D S$ demand system)

alternative development paradigm, 653

American Airlines, 215, 232, 241

amusement, 319, 326, 335, 677

amusement parks, 11-13, 268, 317-319, 664,677

amusement parks, categories of, 320

Antarctica, 775, 843

Antigua and Barbuda, 625, 631

Applied General Equilibrium Models, 472 area life cycle, 8, 10, 212, 225-227, 473, 487, 739

Argentina, 591, 592, 594

*Entries relating to institutions/organizations are listed in both Author Index and Subject Index. 
Aruba, 571, 670

Arunachal Pradesh, 20 21, 22, 705-723

Arunachal Pradesh, growth in its tourism, 721

Arunachal Pradesh, identified tourist spots, 709, 712, 713, 720

ASEAN region, 7, 105

Asia, 4, 70, 106-108, 110, 115, 118, 132, 163, 183, 205, 245, 249, 268, 299, 303, 304, 312, 316, 322-327, 335, 519, 541, 693, 706, 709, 710, 760, 822, 839, 840, 939

Asia-Pacific region, 167, 198, 761

Asian economies, 4, 303, 628

Asian financial crisis in 1988, 179

asymmetric information, 288, 289, 292, 293, 790, 796

attraction development, 259, 263, 267, 275,276

attraction product, 261, 271

attractions, 11-13, 21, 23, 25, 26, 28, 36, 41, 106, 109, 117, 129, 157, 209, 211, 260, 261, 263, 264-270, 272, 273, 275, 278, 317-319, 321, 325, 326, 328-337, 393, 426, 431, 478, 480, 486, 494, 497, $559,564,580,668,673-677,710,714$, 716, 720, 726, 732, 767, 787, 794, 801, $825,848,851,853,855,856,858,859$, $861,871,872,874-876,879,881$

attribution factor, 874

Australia, 3, 12-14, 16, 18, 25, 26, 29, 33, $43,63,66,67,79,81,83,85,87,104$, 107, 110, 116, 131, 168, 183, 215, 268, 283, 287, 299-304, 306-313, 315, 316, $324,325,359,363,371,375,384$, 386-388, 407, 412, 413, 417, 430, 434-436, 439, 443, 445, 447, 463, 465, 466, 472, 473, 475, 477, 482, 490, 492, 493, 495, 500, 502-505, 511-516, 520, 568, 569, 591, 593, 605, 649, 725, 740, 743, 755-758, 761, 765, 773, 783, 785, $787,808,830,842,845,848,849,869$, 871-873, 875, 877, 881-884, 910, 937

Australia's world heritage areas, 866, 876

Australia - expenditure by tourists who visited national or state parks, 875

Australian higher education sector, 301

Australian national parks, 25, 864

Australian tourism, 70, 81, 83, 493, 519
Austria, 12, 66, 85, 103, 198, 649, 664, 681,921

Austrian Airlines, Olympic Airways, 216 aviation services, 10, 221

backpacker, 748, 755, 856

backpacker destination, 694

Bakken, 317, 318

barriers to internationalisation, 146

beach activities, 363

beach and foreshore economics, 444

Beach conservation activities, 450

beach environments, 363

beach expenditure, 458, 461

beach management, 460, 465

beach protection, 14, 377-379, 381, 283, 445,458

beach protection and maintenance, 16, 17 beach recreation, $361,371,387,455,456$, 461, 464

beach replenishment, economic assessments of, 446

beach resorts, 694, 830

beach tourism destinations, 363

beach valuation studies, 361, 365, 367

beach, sun and surf tourism, 11, 361

beaches, 13, 14, 16, 17, 37, 38, 361-364, $367,369,374-376,379,383,386,413$, 444-446, 450, 452, 455, 457, 459-466, 549, 673, 674, 676, 684, 686, 689, 691-694, 700, 747, 775, 776, 791, 796, 828,932

beaches and foreshores, 443, 444, 446, 447, 451, 464, 466

beaches as societal assets, 443

behavioural economics, 6

behavioural economics, experimental economics, 28

Beijing Olympics, 174, 473, 482-484

Belgium, 198, 201, 325, 393, 414, 490, 549,649

Belize, 357, 775

benefits, 7, 15, 17, 21, 27, 30, 34, 38, 115, 129, 164, 196, 203, 242, 244, 261, 265, 269-271, 273, 290, 302, 305, 309, 329, $334,347,356,362,365,368,377,378$, 379, 387, 393, 394, 396-398, 401, 404, 405, 407, 408, 410-413, 417, 419-423, 425, 426, 430, 432, 434, 437, 440, 443-445, 447, 450, 456, 460-464, 476, 
481, 483, 485, 499, 501, 512, 545, 546, $552,555,556,559,565-568,606,617$, 622-624, 652, 656, 657, 660, 663-665, $669,672,678,684,696,722,727,735$, $741,742,749,755,756,775,777$, 781-784, 788-791, 795, 797-799, 803, $804,806,811,817,819,824,835,846$, $847,857,859,863,866,873,883,887$, 899, 907, 911, 912, 914, 916-921, 923, 935, 936

benefits and values, 915

benefits of beach recreation, 452, 455

benefits of conservation, 937

benefits of nature, 937

benefits', 395

Bergson-type social welfare function, 420

Bermuda, 351, 567, 568

Bhutan, 130, 710, 833

Bihar, 123, 126, 127, 129, 130

biodiversity, 25, 27, 126, 439, 705-707, $709,710,721,768,795,813,815,817$, $820,821,830,831,833-835,839-841$, $864,866,884,892,896,912,918,919$, 921, 922, 932, 939

biodiversity conservation, 419, 441, 828,842

biodiversity hotspot, Indo-Burma, 707

biological diversity, 444, 776, 808

biosphere, 711, 814, 820, 839

BonBon-Land, 321, 325

Bophuthatswana, 827

born globals, 144, 160, 167

Botswana, 591, 593

boundaries, 816, 912-914

bounded rationality, 6, 14, 16, 438

Brazil, 125, 169, 203, 473, 476, 487, 549, 591-593, 815, 831

Britain, 13, 70, 131, 223, 260, 264, 290, 309, 318, 327, 549

Brundtland Report, 653

budget constraint, 44, 47, 48, 50, 211, 212, 607,615

bundled holiday package, 214

business, 138, 538

business travel, 84, 219, 237, 238, 499, 524-526, 531, 532, 534, 536, 539

California, 243, 244, 317, 319, 321, 324, 337, 363, 364, 375, 387, 702, 808, 841,939
Cameron Government, 309

Canada, 63, 69, 75, 82, 122, 131, 198, 201, $215,247,254,255,260,270,283,287$, $310,316,327,351,500,519,529$, 591-593, 648, 649, 667, 670, 671, 680,809

canned hunting, 827,826

capacity, 16, 40, 122, 173, 175, 208, 210, 219, 225, 232, 240, 243, 248, 260, 267, $272,273,310,312,313,332-334$, 339-341, 343, 345, 347, 349, 353, 359, 409, 431, 443, 448, 477, 481, 549, 622, $625,635,663,671,672,675,692,734$, 772, 789, 790, 800, 801, 866, 900, 907, $918,919,926$

capacity concept, 806

capital coefficient, 645, 654-656, 658, 659,673

capital formation and inbound tourism, 605

carbon offset schemes, 830

carbon tax on tourism output, 518

Caribbean, 198, 214, 323, 342-346, 351, $355,571,678,755,760,808,815,938$

Caribbean cruise tourism, 358

carrying capacity, 218, 225, 226, 229, 230, 233, 253, 686, 703, 758, 774, 776, 787, 794, 804, 806, 809, 810, 813, 829-831, 905, 910, 911, 931-935, 938, 939

carrying capacity, social, 229, 244 carrying capacity concept, 432, 805, 806 carrying capacity of the planet, 817 carrying capacity, ecological, 805, 907, 931 casino tourism, 750

casinos, 219, 317, 504, 570, 677, 699, 700,827

catch up, 171, 176, 179, 472

catching up by China's hotel industry, 175 causality, 619, 627-632, 634, 635

CBA, 420, 421

CCTs, 837

celebratory phase, 271

Central America, 324, 824

certification, 187, 296, 780, 828, 845, 846

certified tourism products, 855,857

certified, tourism products, knowledge of, 857

CGE, 495

CGE database, 507, 510

CGE model, 511 
CGE model, dynamic, 483, 484, 486

CGE modelling, 506, 520

characteristics, determining the relevant one, 93

consumer demand, characteristics theory of, 211

charter airlines, 9, 198, 200, 213, 214, 216, 217

charter flight tourists, 697

Chhattisgarh, 123, 126, 127, 129, 130

China, 4, 8, 12, 20, 22, 30, 107, 110, 115, 116, 118, 122, 125, 126, 133, 170-190, 203, 232, 284, 294, 303, 304, 308, 310, $312-314,326,335,336,343,473,528$, 541, 602, 633, 649, 651, 677, 704, 714, 725-727, 730, 732-735, 737-740, 755, $766,827,833,868$

China catch up, 8

China Hotel Industry, 189

China, private hotel firm, 179

China's, 21, 126, 133, 171, 172, 174, 177, 182-187, 303, 326, 478, 491, 726, 739,814

China's development, 740

China's domestic tourism, 174, 175

China's economic development, 190

China's economic growth, 172, 189

China's economic reform, 171, 172, 175, 176, 187

China's economic reforms, 174

China's hotel industry, 8, 9, 171, 172, 189

China's international tourism, 173

China's lodging sector, 8, 171, 172, 175, $178,186,187$

China's lodging sector, 177

China's lodging sectors, 8

China's outbound tourism, 180

Chinese domestic hotel firms, 180, 185

Chinese growth, 303, 304

Chinese hotel corporations, 172

choice experiment to value the recreational benefits, 921

choice experiments, 911, 923, 935, 936

choice modelling, 13, 368, 370, 405, 882,923

civil unrest, 121, 123

civil wars, 821

climate change, 17, 66, 85, 361, 362, 365, $383,384,388,389,440,443,444,459$, 460, 462, 464, 481, 506, 520, 765-767,
$772,783,784,815,878,879,881,883$, 884,941

climate change impacts, 464

climate change impacts on beaches, 366

clusters, 19, 89-91, 93, 96, 97, 541, 554,

$555,559,560,562,660,796$

$\mathrm{CO}_{2}$ emissions, 767

$\mathrm{CO}_{2}$-e emissions, 767

coast, 361

coastal, 366

coastal and marine environments, 768, 794

coastal areas, 186, 361, 683, 689, 692,

698,767

coastal assets, 365, 383, 465

coastal cruising, 340,343

coastal economy, 363, 366

coastal infrastructure, 371,377

Coastal recreation, 775

coastal tourism, 222, 361-363, 366, 371,

$388,781,911,938$

coastal tourism assets, 361, 362

coastal tourism, its sustainable and

quality, 692

Colombian drug barons, 821

Columbia, 65, 84, 166, 222, 254, 318, 358, 591, 592, 594

Commission of the European

Communities, 497

common pool, $771,773,777,782,872$,

$883,885,900,905$

common pool resources (CPRs), 885, 891, 893, 899, 900

common property, 787, 792, 793

common property as a source of market

failure in tourism activity, 792

common-property resources, 790, 792

communal management, 885

community-based approach, 824, 837, 838

community-based approach of

ecotourism, 835

community-based co-management of

Ogasawara, 906

community-based ecotourism

management (CBEM), 885

community-based ecotourism management

of Minami-jima Island, 906

competencies and customers, 199

competition from other destinations,

245-247

complete demand systems, $6,35,50,70$ 
Computable General Equilibrium (CGE), 17, 28, 393, 406, 471, 472, 490-493, 495, 570,875

conditional cash transfer programs, 837 conditionalities, 837,838

conservation, $3,5,16,17,23-25,27,30$, $254,364,367,385,432-439,441-444$, 451, 465, 680, 692, 723, 733, 765, 768-771, 773-775, 781, 783, 784, 808, $809,813,815,818-823,825-829$, 835-843, 864, 865, 872, 881, 882, 885, 887, 898-901, 904, 907, 909, 910, 912, 914, 928, 936, 937-940

conservation and ecotourism, 893 conservation issues, 765 conservation and tourism, 3, 939

conservation benefits, 436

conservation NGOs, 832, 835

conservation of natural resources, 435 , 824,908

conservation organisations, $825,836,837$ conservation zones, 821

conservationists, 25, 822, 823, 827, 838 conservative spending, 874 conservative spending measure, 26,874 consolidation, 179, 191, 193, 199-201, 204, $206,357,784,868$

constraints to tourism development in PICs, 755

consumer heterogeneity in tourism, 100 consumer surplus, 397-400, 408, 413, 434, 446, 452, 455, 456, 462, 464, 873, 881,934

consumer surplus and the entry producer surplus, 393

consumer theory of demand, 33, 43 contingent valuation, $277,368,369,377$, $378,383-386,387,405,440,783,785$, $882,849,850,865,866,868,873$

Contingent Valuation Method (CVM), 275, 367, 368, 369, 384,387

Contingent Valuation of Environmental Resources, 387

contribution to foreign exchange, GDP and employment, 744

Convention on Biological Diversity, 833

Cook Islands, 385, 741-744, 746-749, 757, 758,791

Copenhagen, 317, 318, 783
Copenhagen's Tivoli Gardens, 269, 318,323

coral cover, 27, 815, 929, 931, 932

coral reef services, 915

coral reef unit value, 922

coral reef zonation, 920

coral reefs, 768, 782, 796, 820, 834,

911-914, 920, 921, 926, 928-932,

936-940

coral reef, its value 912

corporate social responsibility, 190

corporate social responsibility (CSR)

policies, 752

corporate social responsibility activities, 23,753

corruption, $685,699,825,834,837$

cost and benefit items, their

identification, 393, 402

cost efficiency, 290

cost management, 353

cost of transportation, 39, 238

cost-benefit analysis, 413, 417, 438, 445,

$466,873,925$

cost-benefit analysis, private, 15, 419, 420

Costa Rica, 439, 440, 591-593, 775, 783,

$820,828,840$

costs, 605

costs and benefits, 15, 19, 20, 281, 355,

365, 394-396, 400-402, 404, 406, 407,

420, 605, 609, 788, 842, 867, 934, 935

costs and benefit, quantification, 402

Cote d'Ivoire, 821

creative industries, 275

cross-cultural research, 101

cross-price elasticities, 51, 53, 72, 77, 78,

$81,115,117$

cross-price elasticity, 70, 342

cross-price elasticity of demand, 342, 343

crowding-out effects, 403

cruise industry, 340, 345-347, 356-359,

473, 485, 491

cruise industry, North American, 357

Cruise Lines International Association

(CLIA), 340, 357

cruise ship tourism, 357, 358, 750

cruise ships, 13, 246, 323, 340, 349, 350,

354-356, 537, 768

cruise ships, new, 350

cruise tourism, 13, 339, 340, 355, 357,

358, 492 
cruise-fly, 485

cruising, 13, 196, 231, 232, 234, 339-347

cruising, direct expenditure value, 356

cruising capacity, 343

cruising markets and geography, 342

cruising product, 13, 347, 349

cruising, price elasticity of demand

for, 347

cruising regions, 343

cruising travel agents, 344

cruising's, 343

cultural, 11, 21, 27, 36, 94, 101-104, 107, $125,147,148,155,156,158,161,163$, 164, 184, 264, 265, 270, 275-278, 285, $335,336,367,369,371,383,411,426$, 484, 504, 551, 559, 563, 567, 578, 646, $648,650,652,653,656,660,664,668$, 673, 674, 677, 683-685, 692, 694, 703, 709, 710, 719, 734, 755, 760, 773, 787, $789,799,801,807,814,818,886,887$, 892, 909, 916-918, 922

cultural attractions, 641, 666, 677

cultural development, 260

cultural distance, 155-158

cultural heritage, 39, 222, 278, 568, 673, 689, 705-707, 892

cultural tourism, 260, 664, 677, 710

culturally, 155, 867, 887

culture, interaction of Chinese and

Korean, 737

Cyprus, 473, 479, 485, 489, 568,

591-593, 649

Czech Republic, 670

damages, 800

demand conditions, 38 elasticity, 77, 109

demand for tourism, 3, 5, 6, 7, 34-36, 38, 40, 41, 43, 60, 69, 71, 72, 77, 79, 81, 83, $84,107,108,111,112,115,423,429$, $494,625,766$

demand functions, properties of, 35, 47 demand model specification, 44

demand modelling, system approach, 42, 72

demand systems, 51-53, 56, 60, 61, 64, $67-69,70-72,76,82,84$

demand systems, estimation of, 72, 75

Democratic Republic of the Congo, 823 demographic transition, 816

Demonstration effect, 622, 669, 685

Deng Xiaoping, 176

Denmark, 271, 321, 324, 325, 427, 568, $569,649,739$

density clusters, 96

desertification, 815,840

destination parks, 319, 321, 326, 327, 330, 331,333

developed and developing countries, 154, 286, 297, 590, 623, 636, 647, 652,

660,661

developed countries, 106, 107, 175, 185, 187, 215, 282-284, 287, 290, 293, 419, 596, 598, 599, 646-649, 652, 654, 657, 659, 662, 668, 669, 672, 677, 683, 788, $793,830,833$

developing an attraction, 267

developing countries, 20, 21, 106, 131, 153, 167, 175, 281, 282, 285, 289, 291, 296, 371, 435, 472, 487, 575, 590, 593, 595-597, 599, 622, 625, 636, 646, 652, 654, 658, 661-663, 666, 668-671, 675-677, 683, 702, 788, 789, 793, 797,833

developing countries, management contracts, 154

development, 8-13, 16, 17, 21, 23-26, 28-30, 33, 38, 66, 67, 85, 88, 90, 126, 130, 132, 133, 137, 145, 146, 163, 164, $166,167,169-172,174,176,177,179$, 181-183, 184, 186-189, 198, 204, 214-221, 227, 229, 230, 233, 241, 250, 252, 259, 265, 266, 268-270, 274, 276, 278, 295, 303, 317, 318, 322, 323, 325-329, 333, 335, 339, 340, 345, 349, 356, 361-363, 365, 393, 402, 404, 417-420, 422, 429, 430, 436, 458, 459, 471, 472, 485, 486, 494-497, 498, 500, 506, 519, 520, 543-545, 547, 551, 565, $566,569,572,575,582,587,596,599$, 603, 612, 617, 621, 627, 628, 634, 637-640, 645-652, 654-656, 658, 660-666, 670, 672-679, 680-683, 685, 686, 688, 689, 691-694, 696-698, 700-702, 704, 705, 707, 712-714, 719-722, 725, 727, 730-733, 738, 739, 741, 742, 747-751, 752, 756, 757, 758-761, 765-768, 770, 771, 772, 774, 775, 780, 782-784, 787-790, 795-799, 
801, 804-809, 811, 813, 815, 819, 820, $825,828,829,831,833,836,838,839$, 846, 859, 865-868, 889, 899, 900, 907, 908, 913, 918, 926-928, 930, 933, 936, 937, 939, 940

development process for an attraction, 268 direct visitor impact, 779

discount rates, 421

Disney, 264, 274, 277, 317, 319, 321, 322, $324,326,331,332,335,345,346$, 353,677

Disney Corporation, 260

Disneyland, 268, 269, 277, 319, 321, 326,336

distribution of income, 165, 418, 420, 438,665

dive tourism, 27

dive tourism management, 27, 911

divers and snorkelers, 913, 930

diving impacts, 928

(domestic) travel to Hawaii, 247

domestic tourism, 71, 83, 121, 125, 126, 128, 129, 131, 171, 178, 186, 187, 207, 423, 475, 478, 480, 510, 536, 661

dry parks, 318, 319, 323, 332

duality theory, 45, 63

Dubailand, 335

Dutch disease, 605, 606, 609, 617, 634

e-intermediaries, 204

E-ticketing, 216, 220

eclectic paradigm, 141, 142, 144, 146-148, 153, 164

ECO certification, 845, 848, 849, 852-855, 858-859, 862

ECO Certification, perceived contribution to sustainability, 860

eco-certification, 865, 866

ecological certification of tourism (ECO certification), 25

ecological footprint, 816, 829, 830, 841 ecological impacts, 441, 567, 789, 933

ecologically sustainable development, 465 economic contribution, 505, 517, 520, 683 economic contribution of tourism development, 659

economic development, 3, 5, 20-23, 30, 118, 132, 156-158, 169, 173, 175, 189, 207, 221, 278, 316, 420, 530, 531,
538-541, 544, 568, 604, 618, 619, 633, 636, 637, 654, 810, 869, 883, 886, 912

Economic Development and Tourism, 3, $119,246,248,251,253,254,523$

economic developmental benefits, 645, 646 economic evaluation of alternatives, 418 economic growth, 3, 4, 12, 20, 106, 117,

$122,123,169,172,175,186,249,264$, 269, 277, 303, 308, 309, 494, 506, 519, $555,566,619,621,623-627,630-640$, $645,646,652,653,655,656,658-661$, $663,673,677,678,727,740,741,758$, $807,814,817$

economic impact, 15, 16, 19, 124, 132, 133, 222, 277, 315, 329, 342, 355, 357, 361, 363, 377, 386, 406, 407, 412-414, 437, 472, 475-479, 481-484, 486, 487-492, 501, 502, 505, 517, 541, 543 , $546,548,565,566,568,571,572,741$, $742,754,756,760,783,785,873,875$, $876,878,879,822$

economic impact of tourism, 567, $569,570,757$

economic impact analysis, 14-17, 26, 28, 393, 417-419, 422, 423, 437, 438, 493, $495,505,517,521,875$

economic impact measures, 881

economic impact of cruises, 13, 354

Economic impacts of cultural heritage, 568

economic impacts of tourism, 18-20, 471, $472,492,520,544,545,547,571,645$, $647,653,659,665,741$

economic liberalism development paradigm, 652

economic tourism, 3

economic valuation methods, 871

economic value, 13-16, 21, 25, 277, 369, $374,377,385,386,388,389,422,543$, $548,549,554,566,787,791,867,872$, $873,878,881,882,884$

economies of scale, 9, 147, 148, 157, 197, 228, 339, 347, 350, 353, 424, 430, 620,622

Ecosystem, 939 ecosystem service approach, 911 ecosystem services, 16, 362, 367, 385, 432, 872, 915, 917, 918, 938 ecosystem services, different perceptions and interests, 918 
ecosystem services approach, 27, 911, 912, 914, 916, 937, 940

ecosystem services classification, 916 ecosystems, 777, 779, 828, 892, 907, 912,

914, 918, 920, 921, 931, 937

ecosystem, its ecological succession, 919 ecotourism, 22-26, 30, 357, 419, 653, 705, 707, 719, 722, 723, 740, 748, 769, 770, $773,783,775,784,785,796,799,809$, $810,813,817,818,820,822,826,828$, $830,832,836,838,840,842,864,866$, $867,898,900,908,910,911,926$ ecotourism, its resource-base, 820 ecotourism, real, $818,828,830$ ecotourism and conservation, 828, 837 ecotourism and conservation zones, 827 ecotourism and realpolitik, 832 ecotourism as a tool for sustainable local/regional economic development, 888

Ecotourism Australia, 848, 865 ecotourism development, 834 ecotourism markets, 824, 832 ecotourism, factors determining the possibility, 820

ecotourism, failure of projects, 829 ecotourism, its sustainability, 888 ecotourists, $712,828,831,836$ ecotours, 886, 888, 897, 899, 900, 903, 909 education, 10, 12, 103, 130, 133, 143, 173, 267, 299, 300, 303, 305, 310, 344, 411, 464, 497, 504, 514, 516, 552, 559, 560, $572,620,633,634,646,647,653,671$, $672,678,680,682,703,752,753,755$, $756,760,788,808,854,867,869$, 916-919, 922, 932

ecotours, rules for, 889

Education Australia, 102

education exports, 299, 300, 302-304, 308,313

education exports, growth in, 311, 313 education services, 299, 300, 302, 303, 312,315

education trade, various sectors in it, 307

education tourism, 117, 299, 300, 302, 306, 309, 311, 314

education tourism significance for universities, 304 education tourism, size and significance, 302

educational, 30,687

educational activity, 259

educational tourism, 12

Egypt, 23, 124, 577, 651, 667, 676, 679, 912, 926, 938, 940

Egypt, employment opportunities, 926

EKIG hypothesis (see Export Capital

Good Imports Growth hypothesis)

Elasticities of demand, 54, 79

elasticity of demand, $38,47,77,78$

elasticity of tourism demand, 105

elephants, $824,831,836$

emerging economies, 649, 650, 652, 667,683

empirical results, 76, 105, 107, 117, 152

empirical tourism demand, 33, 34

employment, 3, 21, 33, 35, 122, 270, 293,

$305,313,351,354,355,406,422,428$,

476, 479, 483, 487, 488, 496, 498, 499,

501-503, 505-507, 509, 511, 518, 520,

543-547, 551-555, 557, 558, 565, 566,

$569,600,605,606,611,612,617,622$,

623, 645, 650, 660, 669-672, 685, 696,

703-705, 707, 721, 741, 742, 746, 749, 751-753, 756, 768, 770, 788, 799, 801, $804,806,824,845,877-879$

employment multipliers, 543, 547, 555

energy use, and tourism, 24, 766

energy use, global tourism-related, 767

Engle-Granger test, 112

entrance, 396, 911

entrance fees, 334, 436, 439, 825, 923, 924, 925, 930, 934, 936

entry fees, 431, 433, 435, 441, 872,

873,882

entry formalities, 718,721

entry mode decision, 146

entry-mode decisions in the hotel sector, 156

environment, 3, 24, 30, 96, 102, 103, 106, 133, 154-157, 168, 185, 213, 214, 216-218, 220, 221, 254, 260, 311, 378, $386,388,426,440,460,464,465,480$, $511,590,612,621,639,646,675,694$, 698, 766, 703, 722, 754, 759, 769, 773, 776, 777, 785, 790, 793, 795, 797-801, 803-805, 807, 808, 810, 811, 816, 818, $819,823,826,831,833,837,861,863$, 
864, 866-868, 878, 879, 881-883, $886,887,889,890,893,894,896$, 899, 909, 912, 921, 927, 930, 933, 939-941

environment and tourism, 3, 18

environment, its preservation, 700

environment, its undervaluation, 796, 800

environmental and conservation issues, 23

environmental and conservation

considerations, 765

environmental degradation, costs of, 486

environmental and economic

sustainability, 872

environmental and energy accounts, 471

environmental costs, 363, 393, 396, 798

environmental damage, 355, 385, 486, 659, $675,698,701,770,771,774,796-798$, $802,803,826$

environmental damage, irreversible, 801 environmental degradation, 212, 217, 700, 768, 771, 774, 795-797, 801, 802, 804,898

environmental education, $685,700,886$, 887,930

Environmental Education Forum, 886

environmental externalities, 425, 765, 779, $781,782,804$

environmental externalities associated with tourism, 779

environmental factors, 226

environmental goods, 370, 422, 660, 769

environmental goods in tourism development, 678

environmental goods valuation, 673

environmental impact, 426, 444, 768, 770, $776,780,801$

environmental impact assessment, 218, $686,780,829$

environmental impacts, 27, 426, 627, 653, $675,684,791,792,798,799,807,859$

environmental impacts of tourism, 18 , 474, 480, 492, 500, 779, 789, 809, 842

environmental limits, 657, 659

environmental policy instrument, 765 , $774,780,782$

environmental policy, economic theory of, 778

environmental problems, 10, 771, 772, 778-780, 798, 807 environmental protection, $771,808,833$, $869,881,884$

environmental quality and inbound tourism, 605

environmental resources, 23, 229, 765, 766, 769, 771, 772, 776, 778, 782, 789, $794,800,828$

environmental spillovers, 420, 766

Environments used by tourism, 792

equilibrium models, 15, 493, 519, 572, 627

equity investments, 154

erceived contribution of ECO

Certification, 861

error correction model, 105, 107, 628, 634

essential force: quality scenery and convenient infrastructure, 735

Estimation of Tourism Demand, 67

Etour, 510, 511

Europe, 9, 64, 65, 69, 70, 84, 85, 110, 113-115, 122, 155, 161, 183, 190, 192, 194, 195, 198-200, 203-205, 213-217, $222,223,268,316,318-325,327,330$, 331, 333, 336, 343, 413-415, 429, 575, $584,672,727,744,760,781,814,940$

European, 82, 103, 132, 163, 165

European Union, 215, 479, 586, 670, 678,940

Event China, 314

exchange rate, 108, 110, 304, 307, 309, 312, 474, 475, 480, 483, 487, 582, 631, $634,636,662,681,788,925$

exchange rate effect, 110,115

exchange rate effects, 110

exchange rate policy, 771

exchange rates, 39, 113, 299, 303, 314, 499, 518, 669

expenditure approach, 871,881

expenditure elasticities, $56,59,70,79-81$

expenditure pattern, $65,543,550$, 569,571

Export Capital Good Imports Growth hypothesis (or EKIG hypothesis), 625

export tourism, 37, 619

export-led growth, 619, 623, 627, 628, $635,636,638$

export-led growth literature, 627

exports and economic growth, 623, 636, 637,641

external agents, 795

external economies, 393 
external environmental costs, 425

externalities, 15, 275, 299, 393-396, 402, 413, 420, 429, 607, 621, 627, 766, 770, $772,774,778,790,792,800$

externalities and tourism, 395

externalities as a source of market failure in tourism activity, 791

externalities, common property, 791

externalities, negative, 24, 395-397, 406, $545,675,769,781,782$

externalities, positive, 396, 397, 486, 620, 781,782

externality, 395, 765, 787, 791, 802-804

family parks, 327

FDI, and risk in the destination country, 155

Federated States of Micronesia, 746

FIFA World Cup, 471, 474, 482, 483, 569

Fiji, 20, 22, 23, 123, 124, 471, 473, 482, 491, 570, 741-746, 747, 748, 750-753, $755-760$

Finland, 356, 591-593, 649

firm size, 148, 158, 159

'flagship' enterprises, 329

flag carriers, 9, 213, 214, 216

flags of convenience, 351

flagship species, 825, 829, 831

floating resort, 340

flora and fauna, 126, 218, 379, 675, 705, $711,776,779,791,792,828,917$

fly-cruise, 485

footloose industry, 13

forecasting future tourist flows, 34

foreign capital investment, 658

foreign direct investment, 138, 140, 162, 164, 168, 169, 171, 172, 175-177, 181, 185-187, 576, 601, 680, 755, 759

foreign direct investment by Chinese hotel firms, 185

foreign exchange, 12, 33, 106, 300, 303, $304,506,605,606,616,617,622,623$, $625,639,665,666,668,685,706,741$, $742,747,756,770,788,815,826$

foreign exchange rate, 148

foreign markets, modes of penetrating, 148

foreign operation of tourist accommodation, 748 foreign reserves, 20, 605, 606, 614, 616,617

foreign resources and inbound tourism, 605

foreign students, 12, 299, 300, 302, 305

foreign tourists, 40, 83, 122, 362, 427, 476, 479, 584, 605, 607, 609, 610, 614, 616, $624,665,666,669,693,695,706,713$, $715,717,718,911,912,929$

forest cover, 815

Formula 1 Grand Prix, 407, 408, 410

fragmentation of the value chain, 577

France, 113, 198, 216, 223, 300, 325, $335,336,549,575,603,609,618,640$, 649, 921

franchise agreement, 162, 181, 576

Fraser Island, 770, 781, 910

free-rider problems, 430, 900, 907

French Guayana, 823

French Polynesia, 22, 743, 744, 746-748, 750,791

funfairs, 326

Futurescope, 333

Galapagos Islands, 775, 828, 898, 899

gambling, 504, 516, 700

gambling casinos, 827

game-viewing tourism, 834

GATS (see General Agreement on Trade in Services)

General Agreement on Trade in Services (GATS), 198, 589

German, 113

Germany, 102, 182, 192, 195, 197, 198, 217, 300, 325, 350, 549, 591, 592, 594, 597, 649, 651, 659, 664, 667, 694, 813, 841, 843, 921, 933

global cruise passengers, 341

global cruising, its value of, 341

global economic and financial crisis, 207

global economy, 137, 171, 172, 357, 358, 481,683

global financial crisis, 174, 245, 286, 308, $310,313,322,473,478,491,744,783$

global hotel chains, 243

global market, 132, 146, 180, 188, 199,

286, 344, 347

global marketplace, 868

Global Peace Index (GPI), 131, 132 
global tourism, 4, 22, 165, 304, 600, 601, $646,669,811,865,908,910$

global warming, $24,767,793,830$

globalisation, 138, 146, 156, 296, 297, 324, $478,479,576,577,579,582,600,722$

globalisation in tourism, 19, 575-577, 595

globalisation process, 137

globalised economy, 646

Goa, 20, 21, 170, 356, 683, 684, 686, 688-702, 704

Goa, growth of its tourism sector, 693

Goa, impacts of the tourism growth, 696

Goa Chamber of Commerce and Industry (GCCI), 689

Goa Tourism Development Corporation (GTDC), 691

Goa, process of development, 684

Goa, process of tourism development, 690

Goa, tourism development and social problems, 697

Goa's Vigilant Army, 697

Gold Coast, 14, 17, 29, 30, 252, 325, 372-377, 380, 386, 387, 388, 413, 439, 443-445, 449-452, 453, 455-462,

464-466, 783, 882-884

Gold Coast beaches, 379, 448

Gold Coast City Council, 16, 17, 375, 385, 445, 447, 464-466

good tourism policy, 782

gorillas, 835

government revenues from tourism, 746

government sector, 807

GPAUNIT, 887, 890, 891, 894, 896, 898-900, 903

gravitational attraction, 121

Greece, 161, 168, 207, 223, 224, 351, 591, $592,633,649,671,864$

green products, 854

Gross Domestic Happiness, 833

gross domestic product, 172, 250, 477,

498, 499, 502, 506, 508, 523, 524, 536,

647, 741, 742

Gross World Product, 814

growth of global tourism, 33

Hawaii, 10, 18, 70, 225, 230, 232-238, 240-255, 344, 363, 427, 428, 440, 472, 476, 481, 485, 487, 490-492, 523, 524, $528,530,532,536-538,540,670,791$

Hawaii, domestic travel, 247
Hawaii, visitor arrivals by air, 231

health, 35, 72, 117, 130, 151, 196, 208, 267, 270, 281, 282, 284, 286-290, 292-297, 364, 366, 516, 529, 552, 558, $559,563,620,634,646,647,660,740$, $752,753,830,838,928,930,932$

health insurance, 283, 286, 289-292, 294 health tourism, 12, 281, 287, 296, 566

Heckscher-Ohlin (HO) model, 139

hedonic pricing, 367, 368, 405

heritage and cultural resources, 808

heritage tourism, 277, 279, 709

heritage tourism, adventure tourism, ecotourism, 693

higher education, 299, 301, 302, 304, 305, 307, 311, 313-315

Higher Education Report 2008, 315

Himachal Pradesh, 124

Hong Kong, 110, 116, 176, 182, 260, 295, 316, 329, 349, 541, 605, 649, 677, 727,761

hospital waiting lists, 283, 294

hotel chains, 171, 184, 185, 599

hotel franchising, 154

hotel industry, 154, 164, 168, 177, 179,

181, 189, 428, 601, 789

hotel internationalisation, 160, 167

hotel sector, 8, 137, 152, 154, 160-163, $179,188,689,704,752,864$

hotels, 171

hotspots, 705, 707

hotspots of biodiversity, 820,825

household income multipliers, 543

human nature, 816,836

human population, $23,814,816,831$

hunting, 552, 769, 813, 824, 835, 839

hunting tourism, 826

ICT based services, 677

imagescape, diversity of, 262

imagescape mix, 328

imagescapes, 11, 259-261, 263, 267, 269, $271,272,274,275,276,320,321,326$, 330,335

imagineers, 260, 275, 321, 336

impact analysis, 506

impact of tourists on nature, 902

impact of visitor spending, 877

Impact of tourism, socio-cultural and environmental, 684 
impacts, 15, 17, 18, 22, 205, 206, 218, 226, $229,251,291,339,345,354,361,365$, 398, 400, 407, 412, 418, 422, 439, 441, 443, 445, 446, 458, 460, 462, 464, 471, 472, 482, 484, 488, 493, 495, 499, 512,517

import tariff, 610, 611

import-export indicators of the travel and tourism, 667

inbound tourism, 20-22, 187, 423-425, 430, 477, 512, 514, 516, 585, 605-607, 669

inbound tourism and economic growth, $20,619,620$

inbound tourism, costs and benefits of, 16 inbound tourism, increase in, 20, 512, 513,515

inbound tourism in Queensland, 512 inbound tourism market, 490, 731, 737,738

inbound tourism market development, 737 inbound tourism marketing, 725

inbound tourism to Queensland, 493, 495 inbound tourists, 8, 20, 21, 183, 209, 423, $425,511,610,725,726,731,737$ income and employment multipliers, 437 income and employment multipliers, input-output analysis, 15 income elasticity, 47, 78, 113, 116, 576 income elasticity of demand, 78 incremental internationalisation, 142, 144 India, 4, 7, 12, 20-22, 121-133, 203, 282, 296, 303, 308, 313, 326, 427, 633, 684, 686, 690-692, 695, 696, 700, 702, 703, 705-707, 709-712, 714, 715, 718, 720, $722,723,755,766,815,866$

Indirect Addilog System, 50, 51

India, development processes, 706

India, domestic tourism, 7, 123, 125, 132

India's North-Eastern Region, 715

India's Northeast Frontier, 21

Indian, 316

indirect contribution of tourism, 505, $523-525,528,532,536$

indirect contributions of tourism, 18, 523

Indonesia, 105, 107, 110, 115, 124, 303, 363, 473, 474, 477, 478, 491, 492, 540, $821,833,838$

indoor attractions, 323

industrial attractions, 11, 271 industrial revolution, 318, 577, 600, 814

industry life cycle, 226, 228

information and communication technologies for air transport, 207

information and communication technology (ICT), 9, 223, 662

information asymmetries, 299

information effects on stated WTP, 377

information technology, 8, 191, 205, 222

information treatment design, 378

infrastructure, 366

initial force: pioneers in Korean tourism market, 730

Inner Line Permit (ILP), 713, 714

innovation, 4, 9-11, 13, 161, 175, 191, 194, 204, 205, 228, 229, 233, 243, 249, 252, 253, 259, 264, 265, 276, 277, 317-319, $335,336,358,567,572,622,656,657$, $774,783,883$

innovation and ownership, 263 input-output (I-O) multipliers, 523, 524 Input-Output (I-O) table, 525, 529 input-output analysis, 18, 19, 471, 481,875

input-output database, 508, 518

input-output matrices, 552

Input-Output model, 471, 530, 531, $538,539,541,543,547,548,549$, 553,875

input-output multipliers, 536

input-output table, 19, 485, 487, 495, 507, $551,553,975,877,878$

input-output analysis, 356, 569

insurgent activities, 714

integrated specialisation, 589, 593-596

inter-industry analysis, 3, 17

inter-industry comparisons, 498

inter-industry modelling, 471

inter-industry models, input-output analysis, 17

internal rate of return, 15, 401, 406

internalisation theory, 141, 146

internationalisation, approach to, 145

internationalisation, concept of, 138

international arrivals, 576, 695

International arrivals to Fiji, 745

international arrivals to PICs, 742, 743

international arrivals to the Pacific Island countries, 22 
International Association of Amusement Parks and Attractions (IAAPA), 317

international business, 137, 139, 145, 147, 149, 152, 160-169, 189, 703, 757

international division of labour, 622

international division of production processes, 577,579

international division of tourism production, 19, 584, 588, 602, 679

international division of tourism production (IDTP), 575, 578

international economics and tourism, 3 , 19,618

international fragmentation of production, 575

international fragmentation of tourism production, 579, 590, 600

international hotel chains, 153, 166, 748

international hotel companies, 152, 166, 181, 183

international hotel sector, 153, 155, 163

international new ventures, 144, 160, 163, 167,168

international student market, 300, 301, 309

international students, 12, 300, 301, 304-306, 309-312, 314-316

international tourism, 19, 21, 28, 41-43, 63, 82-84, 122, 124, 131, 169, 206, 208, 213, 221, 222, 349, 472, 476, 478, 483, $488,489,491,521,567,576,590,595$, $598,605,609,616,636,638,640,648$, $652,653,658,662,668,669,675,679$, $680,681,746,747,756,784,788,789$, 868,908

international tourism policy, 605

international tourism receipts, 173, 192, 630, 631, 634, 649, 652, 706, 746, 766,846

international tourist, 9, 21, 22, 29, 35, 94, $126,173,187,414,476,480,576,605$, $606,617,635,661,665,683,693,694$, 696, 706, 726, 727, 766, 787, 789, 793, $828,875,921,924,925,936$

international tourist arrivals, 124, 173, 605, 630, 648, 684, 705, 706, 742,766

international trade in services, 36 international trade theories, 139 international trade theory, 35, 577, 579 international travel, 29, 35-37, 41, 61, 63,65

internationalisation, 8, 9, 19, 137-140, 143, 144

internationalisation in tourism, 152

internationalisation of business firms, 8 , 172, 186

internationalisation of hotel sector in China, 184

internationalisation of service firms, 138, 150

internationalisation of services, 149, 151, 165, 166, 169

internationalisation theories of the firm, 142

internationalisation theory, 141

internationalisation, international experience and degree of, 156, 158, 159 internet, 9, 12, 194-196, 203, 205, 282, 286-289, 292, 294, 295, 331, 373, 385, $559,562,595,599,719,832,837$

internationalisation, network approach, 145

internet searches, 287, 293

investment, 8, 15, 17, 33, 41, 117, 119, $137,141,146,161,163,165-167,169$, $171,172,175-177,181,182,185,188$, 189, 200, 212, 267, 277, 303, 304, 312, $316,319,326,327,329,330,383,393$, 394, 396, 397, 401, 406, 408, 410, 486, 487, 507, 654-657, 719, 734, 810, 825, $845,847,882,884$

investment behaviour, short sighted in the tourism industry, 800

investments, 117, 137, 161, 200, 212, 329,825

Iraq, 207, 245, 473, 477

Israel, 569, 591, 592, 594, 637, 649

Italy, 113, 325, 350, 351, 549, 570, 591-593, 597, 618, 632, 633, 649, 671, 841,921

Italy's, 830

its international specialisation, 19

Jamaica, 591-593, 597, 631, 638

Jammu, 122, 126, 129, 131, 132

Japan, 23, 26, 107, 113-116, 131, 182, 247, 253, 254, 278, 326, 350, 351, 537, 549, 591-593, 646, 648, 649, 651, 652, $667,668,710,718,727,730,761$ 
Japan's National Parks Act, 908

Japanese ecotourism, 886, 888

Japanese ecotourism, criteria of, 888

Japanese ecotourism movement, 886

Japanese ecotours, 887,888

Japanese visitors, 246, 247

Jetstar, 216

Jharkhand, 123, 126, 129

Jordan, 124, 591-593, 631, 638

Kashmir, 122, 124, 126, 129, 131, 132, 696

Kenya, 820, 823, 824

Kerala, 775

Keynesian multipliers, 551, 557, 558

Kiribati, 344, 743, 747, 759

knowledge, 857

knowledge and willingness to pay, 858

knowledge capital transfer, 177

knowledge transfer in hotel

management, 186

Korea, 110, 303, 356, 474, 594, 597, 631, $632,637,725,732-734,736,737$

Korea-China, 910

Korea/Finland, 350

Korean tourists, 22

Korean airline expansion, 734

Korean package group tourists, 726

Korean Wave, 22, 725, 726, 728, 730, 731, 733

Kyoto Protocol, 519, 833

LCC, 212

leakage from the economy, 749

leakages from inbound travel, 668

learning, 97, 143, 145, 146, 165-167, 175,

177, 262, 315, 347, 490, 570, 624, 735,814

leasing agreement, 576

Legoland, 271, 321, 324, 327, 333

Legoland California's, 322

Legolands, 327

leisure tourists, 11, 72, 182

leisure travel, 40, 192, 198-200, 203, 204, 207, 223, 238, 300

leisure travel, leading concerns in Europe, 201

leisure travel value chain, 191, 198, 199

leisure-related energy use, 767

leisure-travel, 202, 213

Leontief model, 525
Leontief's input-output model, 547

less developed countries, 419, 634, 645, $654,664,679$

Liberia, 351, 821, 822

life cycle of Hawaii's tourism industry, 230

lifeguarding services, 463

limits of acceptable change, 806, 810, 829

limits of acceptable change to the environment, 776

'Linear-Approximate' AIDS model, 56, 60

Linear Expenditure System, 33, 50, 51, 67, 68

Linear Expenditure System, Translog Demand Model, 72, 82

lodging, demand for, 9, 175, 188, 427, 428 lodging, demand for in China, 175

lodging, elasticity of demand for, 427

local economic impact, 569

local government, 16, 17, 329, 380, 529, 694, 698, 701, 726-729, 733-735, 889, 891,908

local stakeholders, 818, 825, 835

lodging sector, 8, 171-173, 175, 176, 181, 186, 187

London Olympics, 474, 482, 484

low cost carriers, 9, 194, 202, 212, 223, 224, 599

Lufthansa, 215, 216

Macau, 670

Madagascar, 490, 781

Malaysia, 105, 107, 110, 115, 117, 122, 125, 286, 312, 591, 592, 597, 785, 833

Malaysia, Indonesia, 7

Maldives, 124, 667, 788, 796, 810, 811

Malta, 217, 218, 223, 355, 474, 479, 489, 591-593, 649

man-made capital, 444, 445

management, of beaches, 361

management contracts, $8,154,163,168$, $176,177,181,756$

Maoist rebels, 7, 121, 130

market and non-market valuation, 361, 367

market deregulation, 209, 215

market failures, 15, 23, 24, 141, 429, 439, 607, 770, 790

market power in tourist trade, 38 
market reform in China's lodging sector, 179

market segmentation, 7, 87, 94, 100-104, $171,182,570,739,866$

market segmentation analysis, 6, 87, 88,91

market segmentation strategy, 87,88

market size and growth, 148, 155, 157

marketing and economics, 543

marketing possibilities, 826

marketing strategies, 13, 103, 202,

204, 205, 314, 494, 548, 555, 559,

565,566

mass extinction of species, 813

mass tourism, 117, 197, 242, 362, 371, 413, 571, 653, 697, 769, 826, 888, 889, 907, 927, 928, 931

mass tourism development, 244

Matson Shipping Line, 281, 232

Mauritius, 474, 480, 490, 632, 637, 755

McDonaldization, 342, 349, 358

medical tourism, 12, 281-283, 285-289, 291-295, 297

Medical Tourism Association, 290, 296

medical tourism, concerns about, 289

medical tourist destinations, 289

medical tourists, 12, 281, 282, 284, 285, 289, 290, 293, 296

medical travel, 281, 287

Mediterranean, 69, 82, 84, 202, 205, 214, $217,339,342,343,358,363$

Mediterranean countries, 69, 84, 634, 637

Mediterranean cruises, 340

mega-events, 260, 484

'Mega' park, 328, 329

Mexico, 198, 247, 325, 631, 649, 651, 652, $667,677,782$

Middle East, 322, 323, 335

Millennium Development Goals, UN, 837

Minami-Jima Island, 26, 27, 885, 892-894, 896, 897, 899-903, 905, 907-909, 910

mixed goods, 432, 778

model closures, 509

modernisation development paradigm, 652

monopolistic competition, 30, 272, 273,343

Morocco, 591, 592, 594, 597, 599, 670

Mount Cameroon National Park, 824

mountains, 128, 129, 260, 549, 709, 711, $735,796,818,886$ multi-criteria analysis, 28

multinational enterprises, 141, 181

multinational firms, 140, 622

multiplier effects, 105, 118, 122, 355, 400,

$544,549,554,558$

of tourism, 551, 555, 569

multipliers, 106, 517, 527, 532, 534, 543,

546-548, 551, 553, 554, 558, 565, 568, 570,571

multipliers, normal, 551, 552, 558

museum, 16, 261-263, 268, 271-273, 278, 563,664

Myanmar, 591, 592, 594, 718

National Accounts, 406, 493-496, 501, 540,680

national park, 384, 385, 432, 434, 441, 450, 709, 720, 729, 740, 821, 823, 826, $830,831,841,865,879,881,889,890$, 909, 912, 936, 938-940

national park, Egypt, 27, 911

national park-associated spending, 874 , 880

national parks, 16, 26, 433, 435, 439, 440, $444,570,711,777,824,836,849,878$, $880,883,883,884,891$

national parks, economic value, 25, 871, 883

natural and cultural resources, 906

natural and cultural attractions, 645, 647,732

natural and cultural tourism resources, 548

natural and non-natural remedies, 444

natural areas, 16, 439, 685, 769, 773, 799, $819,826,837,871,873,887$

natural beach capital, 446

natural capital, 17, 385, 444, 445, 938

natural catastrophes, 133, 208

natural ecosystems, 839

natural environment, 26, 244, 358, 653, 684, 771, 776, 794, 795, 802, 871, 873,

886-888, 891, 899, 909

natural environment and culture, 887

natural environment attractions, 882

natural environments, 22, 23, 425, 872,

881,883

nature, $5,8,10,13,21,25,35,37-40,43$, $48,68,294,296,321,567,570,590,593$, 
683, 689, 697, 700, 705, 789, 885, 887, 940, 938

nature and structure of tourist demand, 718

nature conservation, 434, 441, 886, 872, 909, 940

nature rehabilitations and tourist behaviours, 900

Nature Society of Japan's, 887

nature's economy, 458-460, 462, 464

nature-based tourism, 30, 434, 436, 441, 442, 677, 707, 710, 711, 722, 817, 928

nature-based tourism and adventure tourism, 710

neighbourhood effects, 7, 19, 105

neoclassical economic modelling, 19, 423

neoclassical economic theory, 264, 423

neoclassical economics, 6, 14

neoclassical growth theory, 625, 653

neoclassical theory, 48

neoclassical theory of consumer behaviour, 5, 44

net present value, 15, 394, 401, 406, 445

Netherlands, 113, 198, 201, 296, 319, 325, $549,649,922,933$

New Caledonia, 22, 743, 744, 746

New Caledonial, 743

New South Wales, 119, 365, 387, 409, 475, 482, 491, 493, 520, 521, 761, 875

New South Wales Government, 387

New Zealand, 12, 43, 79, 81, 101, 131, 145, 268, 287, 310, 325, 359, 387, 413, 603, $619,640,641,670,680,743,744,746$, $755,758,759,761,785,830,864-867$

Nigeria, 815

Nigerian gangs, 821

Niue, 743,747

non-agricultural, 130

non-market valuation techniques, 367

non-market values, $364,365,383,387$

non-use values, 364, 369, 370, 463

non-use values, economic, 447

Nordic countries, 198, 199, 330, 775

North America, 70, 186, 237, 243, 268, $322,324,327,343,349,600,601$, 744,809

North People's Republic of Korea (NPRK), 735

North-East India, domestic tourist inflows, 716
North-Eastern Region, 711, 720, 722

North-Eastern Region of India, 706, 713, 721,722

North-Eastern States, 706, 712, 715, 721

North-Eastern States of India, 712

Norway, 351, 500, 649

not-for-profit sector, 274, 276

Number of tourist arrivals, 695, 725

ocean acidification, 366

ocean cruises, Major suppliers, 345

ocean cruising, 343, 345, 347, 350, 354

Ogasawara Country Code, 886, 898

Ogasawara Island as a World Heritage site, 907

Ogasawara Islands, 26, 27, 885, 886, 889-893, 895, 899, 901, 903, 906-910

Ogasawara Tourist Association, 891, 893, 909

Ogasawara Village Government, 889, 896

Ogasawara Whale-watching Association, 886,892

oligopolistic, 339, 789

oligopolistic marketing firms, 789

oligopolistic structures within cruise markets, 345

oligopoly, 272, 339, 343

Olympic Games, 260, 397, 571

on-board cruise product, 349

online distribution, 191, 202-204

open access, 13, 771, 773, 774, 778, 782, 792,917

open-access resources, 792, 834

operating force: tourism businesses and their attitude to tourists, 734

opportunity costs, 393, 394, 396, 397, 399-401, 432, 461, 545, 672, 685

optimal tax on tourists resembles, 610

organ harvesting, 285

Organisation for Economic Co-operation and Development (OECD), 35, 301, 520,784

Orissa, 123, 126, 127, 129, 130

outdoor recreation, 205, 273, 277, 375, $444,465,569,809$

output multipliers, 543, 547, 569, 749, 750 overseas tourists, 175, 181, 183, 475, 926 own price elasticities, 53, 59, 70, 71, 76, $77,79,80,116$ 
ownership, 11, 137, 141-143, 147, 160, 177, 179, 181, 182, 184, 190, 199, 237, 259, 260, 265, 267, 268, 270, 274, 276, $324,331,339,345,504,576,578,582$, $585,668,672,674,748,755,778,792$ ownership patterns, 272

Pacific Island Countries (PIC), 20, 22, 741, 756, 758, 785

package holidays, 191-193, 196, 198, 202, 675,789

packaged tour, 242

Pakistan, 131, 633

Palau, 22, 743, 744, 746, 747, 775

Palestine, 125

palm oil plantations, 833

Panama, 351

Papua New Guinea (PNG), 22, 743, 744, 746, 747, 752, 755, 758, 760

Paretian improvement principle, 418, 424

Pareto efficiency, 400, 401

park admissions, 330, 331

park entrance fees, 440, 808

park planning, 317, 330, 909

parks, 13, 165, 260, 262, 268, 269, 274, 276, 321-325, 326-330, 332-337, 379, 383, 432, 434, 441, 450, 774, 785,816

parks, underperforming, 327

Partial specialisation, 589, 593-597, 599, 600

Passenger Mobility Index, 121, 125

Pay-One-Price (POP) admission, 331

Peru, 591-594

philanthropists, 816,837

Philippines, 107, 125, 356, 591, 592, 748, 796

PICs to tourism, its economic significance to PICs, 746

Pilanesberg National Park, 827

pilgrimage tourism, 710

planning, 41, 100, 107, 117, 118, 157, 168, 170, 175, 208, 221, 253, 255, 268, 270, $316,317,327,329,330,332,335,353$, $358,365,367,377,385,413,421,439$, 450, 451, 458, 465, 500, 501, 541, 565, 570, 571, 686, 691, 692, 697, 698, 701, 702, 704, 719, 722, 740, 783-785, 792, $797,798,809,810,881,911,927,928$ poachers, of ivory, 823,824 poaching, $779,823,824,826,831$

Poland, 198, 591, 592, 594, 921

policy incentives, 799

political feasibility, $28,29,813,820,832$

politically feasible, 813,839

poor, 21, 108-110, 113, 125, 126, 129, 420, 487, 626, 665, 666, 700, 817, 920, 929, 933

ports, 344, 349, 353-357

Portugal, 21, 137, 161, 162, 170, 544, 550, 553-556, 559, 560, 565-569, 572, 591, $592,594,633,649,683,690,704$

positive environmental externalities, 781

Posner's principle of justice, 419

poverty, 18, 23, 133, 292, 358, 473, 487, 490-492, 620, 648, 652, 742, 759, 809, $822,836,842$

poverty alleviation, 133, 660, 681, 741,760

preference heterogeneity, 911, 924

'pre-packaged' holiday offer, 202

price elasticities, 54, 56, 57, 59, 63, 64

primary, 46, 144, 172, 174, 175, 183, 184, 186, 207, 221, 224, 292, 374, 388, 427, 467, 481, 499, 507, 511, 514, 546, 560, $621,654,665,670,673,679,692,720$, 726, 755, 773, 788, 848, 869, 874, 876,916

primary education, 561

private sector, 265, 270, 274, 289, 292, 393, 394, 403, 406, 498, 521, 546, 691, $692,720,798,808,886$

producer surplus, 399,400

product differentiation, 13, 37, 103, 346, 349,353

product life cycle, 170, 225, 253, 639

product life cycle theory, 140

prostitution, 685, 697, 806

protected areas, 16, 25, 26, 431-435, 439, $441,549,697,774,806,817,821,825$, $831,832,841,869,871-873,875,876$,

$878,881,882,884,926,938$

psychological economics, 6, 28

public bad, 793

public decision-makers, 411

public economics, 3, 5, 14, 16, 417, 418, 438,520

public education programs, 797

public finance, 29, 417, 438, 617 
public goods, 275, 387, 430, 433, 673, $674,765,772,774-776,778,782,787$, $790,791,793,813,821,839,872$, 884,917

public ownership, 770 public policies, 20, 170, 417, 438, 699, 704 public policy, 316, 388, 423, 467, 499, 683, 732,840

public sector, 11, 24, 170, 263-266, 269, 270, 272-274, 276, 292, 329, 393, 394, 406, 440, 571, 699, 704, 725, 798 pull force: change in tourism supply, 733 pure public good, 431 push force: Government support and aggressive marketing, 732

Qantas, 215, 216, 473

quasi-private good, 16, 24, 431, 777, 793

quaternary activities, 671

Queensland, 3, 14, 16-18, 30, 132, 281, 299, 304, 306, 314, 316, 388, 417, 435, 441-443, 445, 450, 460, $465,466,511$

Ras Mohammed National Park, carrying capacity, 931

racism, 817

random utility method, 367, 369

realpolitik acceptable, 813,839

recreation, $13,16,35,36,70,71,128,208$, $362,363,366-369,377,379,383,384$, $386,388,432$

recreation and tourism, 361, 371, 882, 894

recreation assets, 361, 383

recreation economy, 362

recreational activities, 4, 364, 460, 791,805

recreational returns, 443

recreational surfing, expenditure on, 373,374

recreational surfing, economic value, 371 recreationists, $11,13,14,16$

Reducing Emissions from Deforestation and Forest Degradation (REDD), 838 reef ecosystems, 27, 440, 936, 939 reef services, 911, 914, 917, 919 reef sites, determinants of demand, 930 reef-building corals, 366, 919, 920 reefs, 366, 768, 914, 918, 919, 929, 937 regeneration and property development, 269

regional development, 209, 414, 501, 543, 567, 671, 910

regional economic impact study, 543

regional economic impacts of national

parks, 26

regional economic issues, 18

regional economics, 123, 371, 545, 740,

$878,881-883$

regional economy, 524, 543, 661, 875

regional input-output model, 551, 569

regional tourism, 703, 740

regional tourism economic impact

analysis, 520

regional tourism satellite accounts, 519

reinvestment, 217

relational factors, 159

remote state of India, 705, 707, 719, 721

repeat visits of tourists and recreationists,

13, 249, 259, 270, 271, 334, 335

resort park, 326

resource consumption, 816

restricted area permit (RAP), 713

returns from, 11, 430, 461, 499, 788

revealed comparative advantage (RCA),

$19,579,588,591,592,600,601$

revealed comparative advantage

index, 575

revealed preference, 373

revealed preference methods, 370,465

revenue management, $278,331,339,340$,

$346,353,358$

rhinos, 823, 824, 841

Ricardian model, 579, 583, 601

river cruises, 340, 343, 346, 350

Rodriques, 824

roller coasters, 324, 332

Romania, 355, 640

room taxes, 417, 426-428, 430, 439, 441

Rotterdam Demand System

(RTDS), 51, 52

rural farm stays, 775

Russia, 203, 326, 346, 349, 351, 633, 822, 921, 933

Ryanair, 215, 216, 219, 439

safe minimum standard, 829,830

Samoa, 741-744, 746, 747, 750-752, 757, 759, 918, 939 
Schumpeter's theory, 264

Scotland, 271, 474, 489, 810

scuba diving, 363, 440, 911, 933, 938, 926, 938

Sea World parks, 264

sea-level rises, 767

seasonality, 67, 68, 72, 73, 83, 208, 214

seasonality of tourism, 736

security, 121, 130, 218, 220, 221, 354, 484,

$552,647,821$

security problems in nature reserves, 823

segmentation, 6, 87-93

segmentation model, 544, 549, 554, 566

segmentation research in tourism, 88, 90

segmentation studies, data-driven, 90 ,

94, 104

segments, 544

segments of the tourist industry, 3, 10

segments of visitors, 555

self-imposed management, 885, 892

self-imposed management (SIM) rules for

managing ecotours, 892

self-imposed rules, 26,893

self-packaged holiday, 203

sensitivity analysis, 18, 410, 485, 486, 880

Severe Acute Respiratory Syndrome

(SARS), 245

Six Flags, 321, 325, 327, 335, 337, 677

Social Accounting Matrix (SAM), 476

social and cultural environment, 794

social cost benefit analysis (SCBA),

14-16, 28, 394, 395, 400, 405, 417,

418,422

social impacts, 650

socio-cultural, 686

socio-cultural and environmental impacts, 357,683

soil erosion, caused by ecotourists, 904

South Korea, 592

State of Hawaii Department of

Business, 240

stated preference methods, 384, 873

strategic developments, 223

Sunshine Coast Regional Council, 460

sustainability, 24, 25, 411, 421, 422, 425,

486, 490, 637, 680, 682, 683, 686, 692,

722, 756, 765, 779, 787, 789, 796, 799,

$819,835,842,847,854,859-861,863$,

$869,883,888,928,936$

sustainability, boundaries of, 829 sustainability concept, 653

sustainability development, 654

sustainability issues, 16, 314, 423, 425, 740

sustainability issues in tourism, 769

sustainability of ecotourism, 819

sustainability of tourism, 24, 429, 766,

$805,806,810,841$

sustainable development, 444, 481, 549,

603, 680, 702, 705, 741, 758, 774, 783,

$789,790,801,802,810,817,819,840$,

864, 908, 939, 941

sustainable development in tourism, 884

sustainable industry, 769

sustainable tourism, 787, 883

sustainable tourism in Ras Mohammed, 935

taxation, 605

Thailand, 117, 591

the budget constraint, 78

the changing role of travel agents, 193

the tourism sector, economic

potential, 693

theme park, 263, 277, 319, 325, 326, 328,

330-332, 336, 337

theme park development, 319, 324

theme parks, 260, 264, 271, 278, 317,

324-327, 329, 346, 396, 580, 827

tigers, 827

total economic, 441, 462, 505, 544, 546,

565,841

total economic benefits, 548, 554

total economic impact, $549,554,555$

total economic value, $363,364,367,872$

tourism, 10, 429, 524, 619, 701, 782, 854

tourism, a predatory industry, 828

tourism carrying capacity, 244

tourism carrying capacity in Goa, 692

tourism, characteristics of, 35,40

tourism, economic value, 872,881 ,

883,884

tourism, economic values, 881

tourism, growth of, 4, 229, 235, 247, 427,

$623,694,703,799,801$

tourism goods, as mixed goods, 777

tourism, its economic vulnerability, 124

tourism, its effects on the balance of

payments, 666, 669

tourism, direct economic benefits, 546

tourism, direct economic contribution, 502 
tourism development, its economic contribution, 523, 540, 883, 884

tourism consumption, direct and indirect import content, 535

tourism contribution, $650,655,658$

tourism demand, 505

tourism demand, its modelling, 35, 40, 42, $72,75,82$

tourism demand, measures of, 40

tourism demand econometric issues, 67 , 69,74

tourism demand, determinants of, 34, 38

tourism demand, empirical studies of, 69,79

tourism development, 570, 683

tourism development, challenges for, 711

tourism development, constraints to, 699

tourism development, impacts of, 684, $692,794,828$

tourism direct economic benefits, 546

tourism development in Zhangjiajie, 726, 727,729

tourism development, its effects on the environment, 24, 794

tourism education, 224, 700

tourism externalities, negative, 774

tourism firms, 137

tourism impact, 911

tourism in the Central Region of

Portugal, 19, 543

tourism industry, 756, 796

tourism industry, its long-term viability, 801

tourism market segmentation, 91, 96

tourism policy, 765

tourism prices, measures of, 41

tourism products, demand for, 817

tourism production processes, breaking up of, 600

tourism production, dynamics of the international division of, 590

Tourism Research Australia, 875, 876, 880,884

tourism resources, 405, 424, 731, 737, 770, $771,778,787,799,872$

tourism satellite account for Hawaii (HITSA), 528

Tourism Satellite Accounts (TSAs), 5, 17, 18, 406, 494, 496, 497, 487, 489, 493,
511, 520, 523-526, 528, 529, 531, 532,

$536,537-539,540,544,670,875,880$

tourism's international splitting up, 578

tourism's, value added changes,

international splitting up, 578

tourism-led growth, 20, 619, 636

tourism-led growth (TLG) hypothesis,

$620,630,635,637-639$

tourist developments, 28, 417, 418, 420,

426, 429, 438, 788

tourist expenditures, import content, 525, 528

tourist intermediaries, 9

tourist magnetic site, 121, 123

Tourist Regional Multiplier Model, 547

tourist spots, 129, 130, 709, 710, 712, 713, $717-719$

tourists' expenditure, $527,871,872$

tourists' motivations, 348

trade in tourism, 37, 38, 587, 588

transaction and communication costs,

$578,580,600$

transaction cost theory, 140, 141, 166

Transaction costs, 4, 24, 141, 162, 166, $419,804,854$

Translog, 53-57, 59, 61, 63, 67, 76

translog demand model, 33

translog demand system, 53, 64, 68, 69

transport, cost of, 41, 43

transport, demand for, 208

transnational companies, 748, 750, 756

transplant surgery, 284

transport and communications

technologies, 594

transportation, cost of, 39, 238

transport sector, 207

transport sector, and tourism, 207

transport services, 124

transportation, 36, 39, 41, 43, 70, 71, 109,

144, 151, 193, 214, 222, 226, 229, 230,

232, 233, 237, 240, 249, 251, 252, 344,

476, 479, 487, 494, 528, 530, 531,

$533-535,538,575,576,581,597,600$,

$666,677,699,700,718,719,787,794$,

$825,889,938$

travel, 538

travel agencies, 191, 193-195, 197, 198,

200, 201, 203, 204, 219, 559, 562, 578,

$590,676,728-730,733,734,737,738$

travel agencies, changing role of, 196 
travel agent, 9, 122, 185, 191, 192, 194-196, 205, 216, 220, 233, 580, 675, 730,926

travel and tourism, contribution to GDP, 766

travel and tourism, Determinants of demand, 38

travel and tourism giants, 200

travel and tourism intermediaries, 9, 191-193, 204, 205

travel and tourist agents, 9

travel cost analysis, 865,873

Travel Cost Method, 13, 367-369, 405, 409, 447, 452, 462

Travel costs, 43, 110, 369, 405, 409, 413, 448, 452, 462, 734

travelling for treatment, 281

trends, 7, 22, 59, 166, 168, 171, 205, 223, 228, 239, 240, 244, 252, 253, 269, 288, $296,300,314,317,329,358,579,639$, $715,740,816,887$

Trends in tourist inflows, 717

tribes, 121, 705, 707, 710, 714, 833

trolley parks, 318

trophy hunting, 826, 834

TSA/RMF, 494, 496, 497, 520

TSAs (see Tourism Satellite Accounts)

Tunisia, 591-593, 631, 635-637

Tuvalu, 743, 747, 757, 759

UK, 12, 30, 64, 69, 79-81, 83, 102, 107, 113, 116, 198, 760, 783, 784, 808

UK demand for tourism, 69, 75

Ukraine, 355

United Arab Emirates, 323, 646

United Liberation Front of Assam (ULFA), 714

United Nations World Tourism

Organization, 223, 520

United States, 63, 69, 75, 79, 81, 82, 116, 122, 124, 183, 192, 198, 207-209, 215, 228, 229, 241, 252, 255, 300, 303, 310, 371, 428, 465, 467, 497, 508, 523-526, $529,540,591-593,600,618,646$, 648-652, 666, 667, 669, 809, 810, 833,892

Universal Studios, 264, 271, 321, 322, 327,335

unsustainable development, 657 unsustainable environmental consequences, 771

UNWTO (see United Nations World Tourism Organisation)

UNWTO TSA:RMF, 525

Uppsala Model, 142-144, 153

urban environment, 794, 795

Uruguay, 594, 597, 631, 637

US foreign outbound travel data from US Department of Commerce, 248

USA, 9, 30, 64, 65, 107, 110, 114, 116, 124, 125, 167, 170, 190, 192, 196, 205, 214, 215, 225, 254, 271, 295, 300, 308, $312,314,316,319,321,323,327,331$, 412, 440, 441, 466, 472-474, 484, 523, $540,549,571,625,640,651,668,718$, $784,810,865,922,924,933,939$

US-China WTO Agreement, 190 use and non-use values, 364,378 user-fees, 430-437, 440

user-pays, 439

user-pays principle, 16, 417, 430, 431 user-pays principle and tourism, 430 utility function, 44-46, 49-55, 59, 60, 62, $486,607,613,614$

valuation methods, 14, 368, 369

valuation of benefits, 403

valuation of cost and benefit items, 393, 403

valuation of environmental resources, 769

valuation of the environment, 465

valuation value (VV), 673, 674

value added chain, 579

value judgements, 417, 438, 932

Vanuatu, 22, 355, 741, 743, 744, 746-748, $751,752,756,758-760$

vertical specialisation', 577

Vietnam, 107

Village Roadshow chain, 325

violence, and tourism, 7, 121, 124, 128-130, 132, 697, 822

visas, 282, 299, 309-311, 582, 585, 606, 676

visitor attendances, 271

visitor attractions, 259, 262, 264, 270,

271, 274, 276, 278, 324, 327, 337

visitors, 544 
volume of domestic tourism, India's

Passenger Mobility Index was

calculated, 125

voluntary rules, 27, 900

voluntary sector, 267,273

Wales, 11, 52, 53, 59, 63, 65, 74, 76, 83, 84, 279, 466, 475, 511, 878, 879, 882,883

Walt Disney Attractions, 322

Walt Disney parks, 260

wanderlust travel, 37, 38, 245

wanderlust travellers, 36

Warner Bros Movie World, 321, 325

waste production, 816

water parks, 317, 322, 323, 325, 326, 332

wealth maximisation, 418

web of life on Earth, 815

welfare, 29, 38, 62, 267, 296, 399, 405, 417-419, 422-424, 429, 431, 437, 438, 440, 475, 476, 480, 487, 605, 606, 608, 610-617, 624, 637-639, 646-648, 654, 742, 754, 756, 767, 784, 790, 791, 798, $810,828,873,914,917,919,924$

welfare benefits, 479

welfare economics, 417, 418, 422, 438, 439, 646

welfare effects of increased inbound tourism, 608

West Bengal, 123, 126-130, 718

Wet Tropics of Queensland World Heritage Area (WTQWHA), 848, 849

Wet Tropics World Heritage Area, 845, $865,868,877$

whale watching, $784,775,824,886,892$, $893,895,896$

whale-watching ecotours, 889

wild goats, 895, 898, 900, 909

wilderness and protected areas, 431

wildlife, 17, 130, 260, 433, 435, 439, 440, 450, 473, 484, 492, 716, 720, 768, 769, $774,783,794,808,818,823-826,871$, $875,882,883,896,897$ wildlife conservation, 840

wildlife parks, 580

Wildlife tourism, 441, 711, 720, 769, 780, 783,784

willingness to pay, $14,274,371,384,387$, 393, 397, 398, 400, 401, 404, 405, 613, $849,853,855,856,857,861,864$, 866,867

willingness to pay for ECO certification, 853

willingness to-pay, 441, 845, 847, 851, 868, 939

World Heritage Areas, 876, 877, 883

World Heritage Site, 22, 26, 126, 130, 265, 716, 725, 885, 906, 907

World Natural Heritage Areas, 725, 726,886

World Tourism Organisation (WTO), 35, 229, 497, 517, 519-521, 523, 540, 603, 617, 680, 692, 704, 760, 788, 809, 811,867

WTO (see World Trade Organisation)

World Trade Fairs, 260

World Travel and Tourism Council (WTTC), 497, 744, 760, 785

Wulingyuan, 22, 725, 726, 740

Wulingyuan Scenic Area, 727

Yugoslavia, 668

Zhangjiajie National Park, 22, 725

Zhangjiajie, annual tourist arrivals, 727

Zhangjiajie, impacts of the Korean tourist arrivals, 736

Zhangjiajie, improvement of tourist supply of tourism facilities, 736

Zimbabwe, 834

'zero fee' policy, 734 